ARTICLE

Received 4 Sep 2015 | Accepted 8 Dec 2015 | Published 2 Feb 2016

DOI: $10.1038 /$ ncomms10406

OPEN

\title{
Transcriptional silencing of long noncoding RNA GNG12-AS1 uncouples its transcriptional and product-related functions
}

Lovorka Stojic ${ }^{1}$, Malwina Niemczyk ${ }^{1}$, Arturo Orjalo ${ }^{2 \dagger}$, Yoko Ito ${ }^{1}$, Anna Elisabeth Maria Ruijter ${ }^{1}$, Santiago Uribe-Lewis ${ }^{1}$, Nimesh Joseph${ }^{1}$, Stephen Weston ${ }^{3}$, Suraj Menon ${ }^{1}$, Duncan T. Odom ${ }^{1}$, John Rinn ${ }^{4}$, Fanni Gergely ${ }^{1} \&$ Adele Murrell ${ }^{1,3}$

Long noncoding RNAs (IncRNAs) regulate gene expression via their RNA product or through transcriptional interference, yet a strategy to differentiate these two processes is lacking. To address this, we used multiple small interfering RNAs (siRNAs) to silence GNG12-AS1, a nuclear IncRNA transcribed in an antisense orientation to the tumour-suppressor DIRAS3. Here we show that while most siRNAs silence GNG12-AS1 post-transcriptionally, siRNA complementary to exon 1 of GNG12-AS1 suppresses its transcription by recruiting Argonaute 2 and inhibiting RNA polymerase II binding. Transcriptional, but not post-transcriptional, silencing of GNG12-AS1 causes concomitant upregulation of DIRAS3, indicating a function in transcriptional interference. This change in DIRAS3 expression is sufficient to impair cell cycle progression. In addition, the reduction in GNG12-AS1 transcripts alters MET signalling and cell migration, but these are independent of DIRAS3. Thus, differential siRNA targeting of a IncRNA allows dissection of the functions related to the process and products of its transcription.

\footnotetext{
${ }^{1}$ Cancer Research UK Cambridge Institute, University of Cambridge, Li Ka Shing Centre, Robinson Way, Cambridge CB2 ORE, UK. ${ }^{2}$ Biosearch Technologies Inc., 2199S. McDowell Boulevard, Petaluma, California 94954, USA. ${ }^{3}$ Centre for Regenerative Medicine, Department of Biology and Biochemistry, University of Bath, Claverton Down, Bath BA2 7AY, UK. ${ }^{4}$ Department of Stem Cell and Regenerative Biology, Harvard University, Cambridge, Massachusetts 02138, USA. †Present address: Genentech, Inc.1 DNA Way, South San Francisco, California 94080, USA. Correspondence and requests for materials should be addressed to L.S. (email: lovorka.stojic@cruk.cam.ac.uk) or to A.M. (email: a.m.murrell@bath.ac.uk).
} 
T he mammalian genome encodes many long noncoding RNAs (lncRNAs) that play pivotal roles in gene regulation during development and disease pathogenesis ${ }^{1-3}$. LncRNAs can regulate gene expression through transcriptional interference $(\mathrm{TI})^{4,5}$ or through the RNA product itself ${ }^{1,2}$. TI occurs when the act of transcribing a gene directly interferes with the transcription of an adjacent gene in cis, either at its initiation site or at an essential cis-regulatory element ${ }^{6,7}$. Transcription of lncRNA has been shown to interfere with the transcription of adjacent genes by altering the binding of transcription factors ${ }^{8-10}$ and/or RNA polymerase II (Pol II $)^{11}$, nucleosome remodelling ${ }^{12}$ or inducing changes in histone modifications ${ }^{13,14}$.

So far, the strategies for demonstrating TI in mammalian cells have relied upon genetic engineering methodologies that remove the promoter region or insert premature transcriptional termination sites within the transcriptional unit $^{4}$. An alternative approach would be to leave the DNA template intact and directly target the lncRNA with small interfering RNAs (siRNAs). It is widely perceived that RNA interference (RNAi) is a cytoplasmic pathway for post-transcriptional gene silencing ${ }^{15}$. However, RNAi-based technology has been used to posttranscriptionally deplete a lncRNA Kcnq1ot1 in the nucleus and demonstrate that this IncRNA product is not required to maintain imprinting of adjacent genes ${ }^{16}$. RNAi has also been shown to induce transcriptional gene silencing (TGS) ${ }^{17-19}$. siRNA-directed TGS can lead to epigenetic changes such as DNA methylation and histone methylation at the target promoters ${ }^{17,20-22}$. In addition, promoter-targeting siRNAs can induce TGS by blocking the recruitment and the activity of Pol II (refs 23-25). In all these cases, Argonaute proteins (AGO1 and AGO2) were shown to be key players of TGS ${ }^{21,24,26-29}$. Finally, it has been shown that siRNAs can be used to knockdown small-nuclear ncRNA $7 \mathrm{SK}^{30}$. These observations prompted us to investigate whether siRNAs can be used to inhibit lncRNA transcription and to explore the functional consequences of this process. In particular, we postulated that by targeting different regions of the lncRNA, we could uncouple the act of transcription from the function of the transcript. This would enable us to investigate how lncRNAs regulate adjacent genes in cis through TI. As a model, we used the imprinted tumour-suppressor DIRAS3 locus, where we have recently characterized a novel lncRNA known as GNG12-AS1 (ref. 31). GNG12-AS1 is transcribed in an antisense orientation to DIRAS3 and its neighbouring non-imprinted genes GNG12 and WLS (Fig. 1a). We have shown that this lncRNA is allele specifically silenced in cancer cell lines depending on the imprinted state of DIRAS3 (ref. 31). DIRAS3 (also known as ARHI and NOEY2) is a Ras-related imprinted tumour suppressor involved in the inhibition of growth, motility and invasion via several signalling pathways including RAS/MAPK, STAT3 and PI $3 \mathrm{~K}^{32}$. DIRAS3 is downregulated in $70 \%$ of breast and ovarian cancer ${ }^{33-35}$, and its loss of expression correlates with cancer progression and metastasis $^{34,35}$. The mechanism responsible for DIRAS3 downregulation to date involves different epigenetic mechanisms and loss of heterozygosity ${ }^{32}$. We hypothesized that TI by GNG12-AS1 could represent an additional layer of regulating DIRAS3 dosage.

Here, we demonstrate that GNG12-AS1 can be transcriptionally silenced with siRNAs complementary to a region proximal to its transcriptional start site (TSS). The transcriptional silencing of GNG12-AS1, which is mediated by AGO2, leads to upregulation of DIRAS3 transcription in cis, indicating a function in TI. In contrast, targeting GNG12-AS1 at the $3^{\prime}$ end did not affect its nascent transcription, but reduced the lncRNA through post-transcriptional gene silencing and, importantly, did not affect DIRAS3 transcription. We further show different phenotypic effects in cell cycle and migration depending on whether we target the $5^{\prime}$ or $3^{\prime}$ end of GNG12-AS1. Altogether, our results demonstrate that strategic targeting of siRNA to different regions of an IncRNA can enable the discrimination between functions related to its active transcription and that of the RNA product.

\section{Results}

GNG12-AS1 is a stable lncRNA localized in the nucleus. In this study, we used three non-cancer cell lines (HB2, HS27, MCF10A), which we have previously shown to have normal imprinted DIRAS3 expression, and breast cancer cell lines (SUM159, MCF7), where loss of DIRAS3 imprinting leads to biallelic expression (SUM159) or biallelic silencing of DIRAS3 (MCF7). The non-cancer cell lines expressed GNG12-AS1 from both alleles, whereas the cancer cell lines expressed GNG12-AS1 from one allele ${ }^{31}$. We confirmed the relative expression of DIRAS3, GNG12, GNG12-AS1 and WLS in these cell lines (Supplementary Fig. 1a). Actinomycin D chase experiments indicate that GNG12-AS1 is a stable lncRNA with a half-life between 20 and $25 \mathrm{~h}$ (Supplementary Fig. 1b). DIRAS3 expression remained unchanged in HB2 and increased in HS27 when cells were treated with Actinomycin D, suggesting an inverse relationship between DIRAS3 and GNG12-AS1 transcription. Despite being a stable IncRNA, GNG12-AS1 has a low transcript volume (20-80 molecules per 100 cells; Supplementary Fig. 1c). Expression analysis following cell fractionation indicated that GNG12-AS1 is localized within the chromatin (Fig. 1b), similar to MALAT1, a lncRNA known to be associated with chromatin ${ }^{36}$. Using single-molecule RNA fluorescence in situ hybridization (RNA FISH; as described in ref. 37), we confirmed that GNG12AS1 is nuclear in HB2 and SUM159 cells. Exonic probes complementary to all exons showed that GNG12-AS1 transcripts accumulate in the nucleus in discrete foci in 19\% of SUM159 and 25\% of HB2 cells (Supplementary Fig. 1d).

Intronic RNA FISH probes complementary to the first intron of GNG12-AS1 were found to co-localize with the exonic signals in about two-thirds of cases where both probes were present in the same cell (6.8\% in HB2 cells; $2.8 \%$ in SUM159 cells; Fig. 1c). As intronic probes are usually indicative of nascent transcription and exonic probes can detect both primary and mature transcripts, their co-localization may indicate that the processed transcript remains at its site of transcription, which would fit with GNG12-AS1 being co-transcriptionally spliced, as previously reported $^{31}$. However, as we also see separate signals for exonic and intronic probes, it is likely that some GNG12-AS1 also accumulate at other sites in the nucleus away from its site of transcription (3.3\% in HB2 cells; 1.7\% in SUM159 cells; Fig. 1d). A caveat to the interpretation of FISH data is that intronic and exonic RNA FISH probes might not hybridize to nascent and mature lncRNA transcripts in a mutually exclusive way. Nascent RNA contains exons and the mature lncRNAs may be present as unspliced isoforms.

In SUM159 cells, the intronic probe mostly produced a single FISH signal (13\% of cells being monoallelic, Supplementary Fig. 1e), which fits with previous pyrosequencing data reporting allele-specific GNG12-AS1 expression in cancer cells ${ }^{31}$. By contrast, in HB2 cells, even though pyrosequencing showed an allelic ratio of $50 \%$ (ref. 31 ), we observed cells with both single and double intronic signals (8 and 6\% of cells, respectively; Supplementary Fig. 1e), suggesting that GNG12-AS1 is heterogeneously biallelic and randomly monoallelic at the cellular level in HB2 cells. Together, these data confirm that GNG12-AS1 is a stable IncRNA that is present in the nucleus at distinct foci. 
a

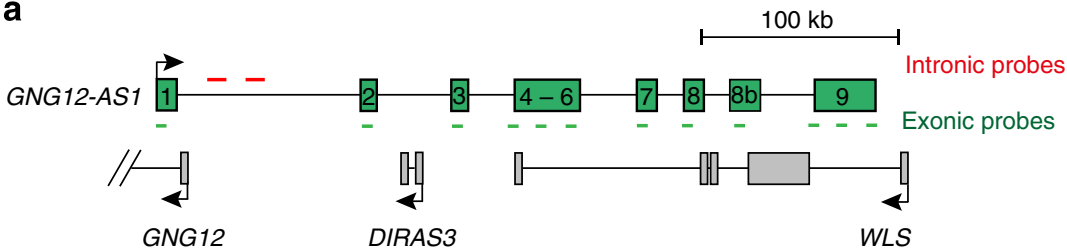

b
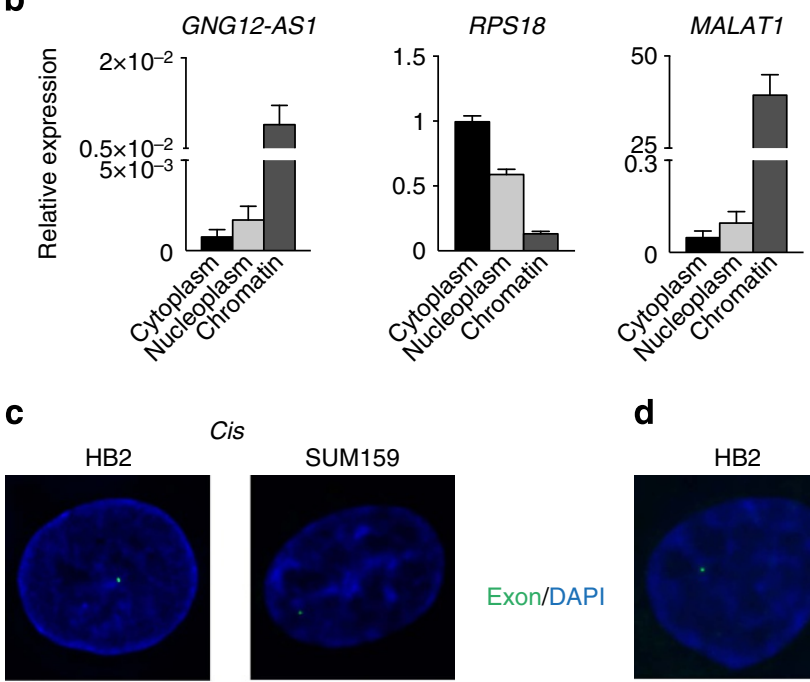

Cis
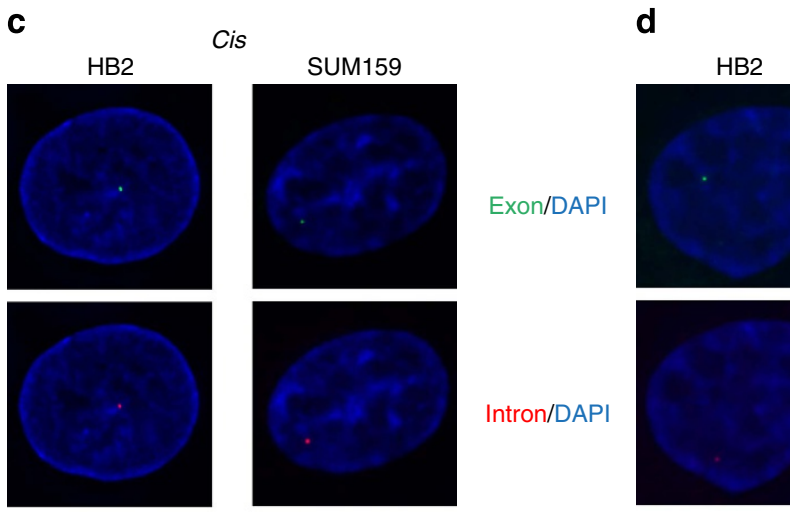

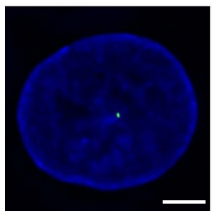

$6.8 \%$

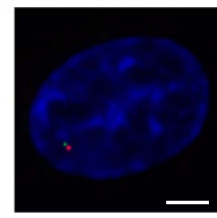

$2.8 \%$
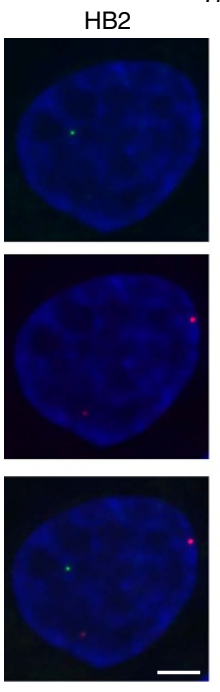

$3.3 \%$
Trans

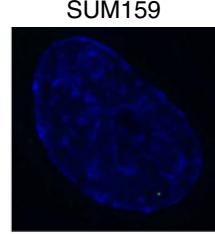

Merge
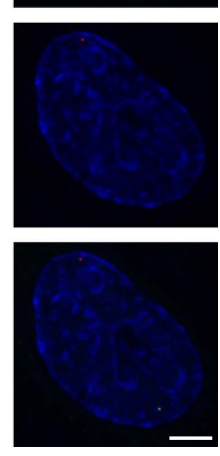

$1.7 \%$

Figure 1 | GNG12-AS1 is a stable IncRNA in the nucleus. (a) Schematic representation of the GNG12-AS1 genomic locus (chr1: 68297971-68668670, hg19) relative to GNG12, DIRAS3 and WLS. The arrows show the direction of transcription. GNG12-AS1 exons are in green and are numbered as previously reported ${ }^{31}$. Probes used for FISH are marked. Intronic, labelled in red identify the site of transcription. Exonic, labelled in green, mark mature transcripts. (b) RNA distribution from the cytoplasm, nucleoplasm and chromatin in SUM159 cells as quantified by qRT-PCR. RPS18 and MALAT1 are positive controls for the cytoplasmic and chromatin fraction, respectively. Note enrichment of GNG12-AS1 in the chromatin fraction. Relative RNA levels are standardized to the geometric mean of GAPDH and $\beta$-actin. Error bars represent the s.e.m. values of three independent experiments. (c,d) Co-localization of exonic (green) and intronic (red) GNG12-AS1 probes in HB2 and SUM19 cells by single-molecule RNA FISH. GNG12-AS1 associates with its site of transcription (2.8\% in SUM159, 6.8\% in HB2; cis, c) but is also present at the other sites in the nucleus (1.7\% in SUM159, 3.3\% in HB2, trans, d). The numbers represent the percentage of cells positive for exonic and intronic FISH signal ( $n=132$ for HB2; $n=176$ for SUM159). The nucleus was stained with 4,6-diamidino-2phenylindole (DAPI). Scale bar, $7 \mu \mathrm{m}$.

TI between GNG12-AS1 and DIRAS3. To investigate the role of GNG12-AS1, we depleted GNG12-AS1 using siRNAs (Fig. 2a). GNG12-AS1 has multiple isoforms ${ }^{31}$, thus we designed siRNAs against several different exons and found that siRNA targeted to exons 1,5 and 7 reduced GNG12-AS1 by $50-70 \%$ in HB2 and SUM159 cells (Supplementary Fig. 2a-c). These exons are common to all of the known splice forms of GNG12-AS1. Using several primer sets to capture the various GNG12-AS1 isoforms, we confirmed that siRNAs against exons 1 and 7 efficiently knockdown GNG12-AS1 (Supplementary Fig. 2d). We further confirmed that the knockdown occurred in all cellular compartments (Supplementary Fig. 2e). Thus, we are able to deplete GNG12-AS1 in the nucleus with siRNA.
RNA FISH analysis in SUM159 cells showed that although siRNAs to either exon 1 or exon 7 effectively ablated GNG12-AS1 detectable with exonic probes, siRNA targeting exon 1 reduced GNG12-AS1 detectable with intronic probes (nascent transcripts) more efficiently than siRNA to exon 7 (Fig. 2b). These results indicate that although both siRNAs can efficiently reduce GNG12-AS1 levels in different cellular compartments, siRNA complementary to the $5^{\prime}$ end of GNG12-AS1 may preferentially inhibit its nascent transcription.

We next asked whether depletion of GNG12-AS1 affects the transcription of its neighbouring genes. We analysed the expression of GNG12, DIRAS3 and WLS after depleting GNG12-AS1 with siRNAs against exon 1 and exon 7. DIRAS3, but not GNG12 or WLS, was upregulated after targeting exon 1 of 
a

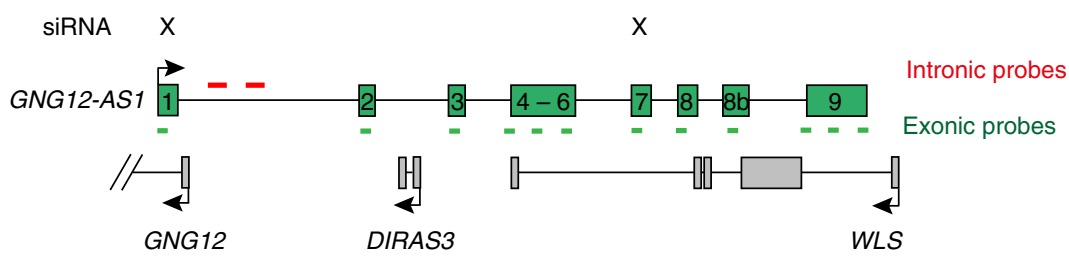

b

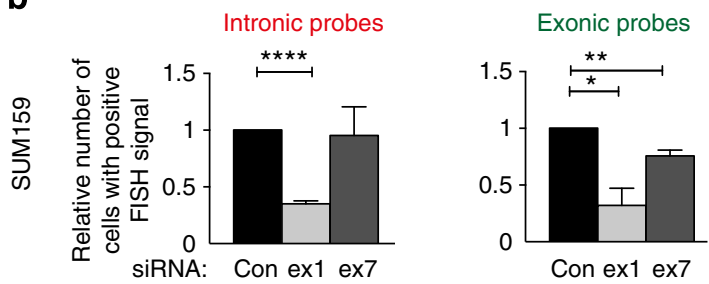

C
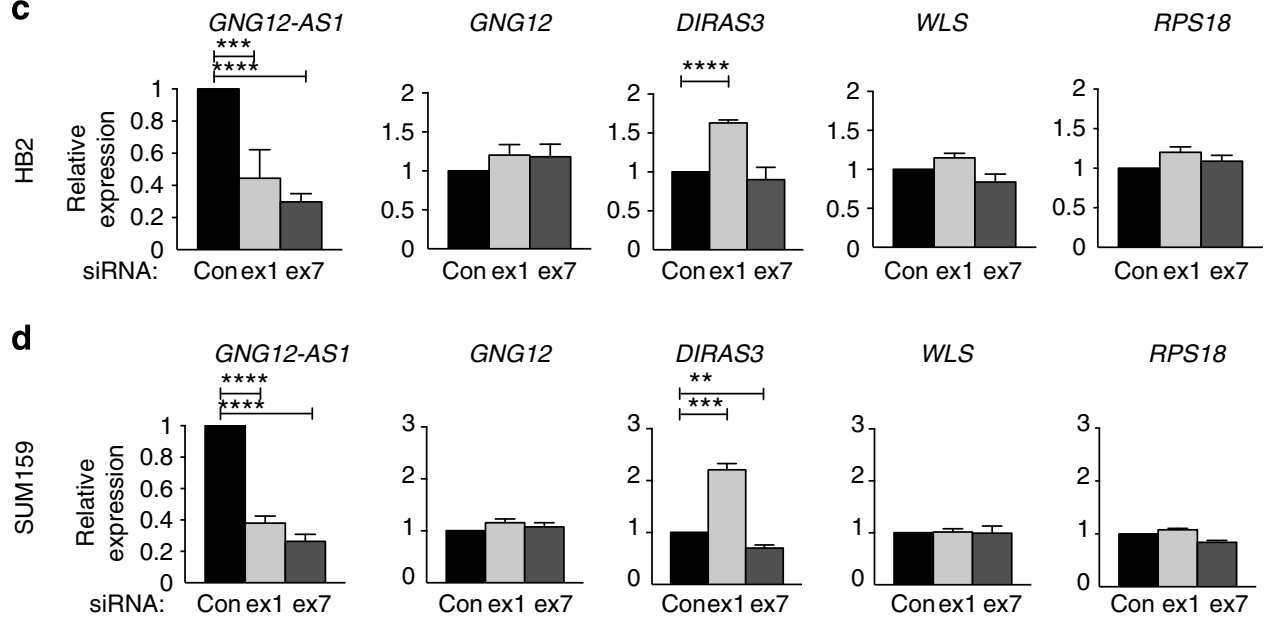

Figure 2 | Transcriptional interference by GNG12-AS1 regulates DIRAS3. (a) The GNG12-AS1 locus where X indicates the exons targeted by siRNA. (b) Changes in single-molecule RNA FISH signals after treating SUM159 cells with siRNA directed to exon 1 or exon 7 of GNG12-AS1. Changes in nascent transcription after exon 1, but not exon 7 siRNA, of GNG12-AS1 were found using intronic probes (left panel). Both siRNAs decreased the number of exonic FISH signal (right panel). Cells positive for intronic probes include cells with both mono and biallelic signal, and cells positive for exonic probes include cells having one or more dots. The number of cells positive for RNA FISH signal is presented relative to control siRNA (control siRNA $=454$ cells; exon 1 siRNA $=429$ cells; exon 7 siRNA $=257$ cells). (c,d) siRNA-mediated knockdown of exon 1 ( $5^{\prime}$ targeting) and exon 7 of GNG12-AS1 ( $3^{\prime}$ targeting) in HB2 (mammary epithelial cell line, c) and SUM159 (breast cancer cell line, d). Similar results were obtained in two additional cell lines (Supplementary Fig. 3a,b). DIRAS3 is upregulated only when the $5^{\prime}$ end of GNG12-AS1 is targeted by siRNA directed to exon 1. For all the graphs, expression levels of DIRAS3, GNG12, WLS, GNG12-AS1 were measured by qRT-PCR, normalized to GAPDH or to geometric mean of GAPDH and RPS18 (see also Supplementary Fig. 3c-f), and are presented relative to control siRNA. Primers spanning exons 7-8 were used for GNG12-AS1 expression. RPS18 was used as a negative control gene whose expression does not change upon siRNA treatment. Error bars, s.e.m. ( $n=3$ biological replicates). ${ }^{\star} P<0.05,{ }^{\star \star} P<0.01,{ }^{\star \star \star} P<0.001$ and ${ }^{\star \star \star \star} P<0.0001$ by two-tailed Student's $t$-test.

GNG12-AS1 in four cell lines (Fig. 2c,d and Supplementary Fig. 3a-f). DIRAS3 upregulation was consistent in different cell lines when GAPDH (Fig. 2c,d and Supplementary Fig. 3a,b) or the geometric mean of GAPDH and RPS18 were used (Supplementary Fig. 3c-f). Thus, for further quantitative realtime PCR (qRT-PCR) experiments, we used GAPDH as the reference gene. In a fifth cell line MCF7, despite a $60 \%$ reduction in GNG12-AS1, expression of DIRAS3 could not be reactivated (Supplementary Fig. 4a). In MCF7 cells, DIRAS3 is more hypermethylated at its promoter compared with SUM159 cells $^{31}$. Off-target effects were excluded by the use of additional siRNAs against exon 1 and exon 7 of GNG12-AS1 (Supplementary Fig. 4b). Furthermore, we used the randomized nucleotide sequence (scrambled siRNAs) of exon 1 and exon 7 siRNAs (Supplementary Fig. 4c,d), as well as C911 mismatch siRNA controls $^{38}$, all of which failed to affect GNG12-AS1 and DIRAS3 levels (Supplementary Fig. 4e).
To exclude the possibility of GNG12-AS1 regulating DIRAS3 expression in trans, we ectopically introduced GNG12-AS1 in SUM159 and HB2 cells. Overexpression of the most common splice variants of GNG12-AS1 had no effect on expression of DIRAS3 or the surrounding genes (Supplementary Fig. 5a,b). As DIRAS3 is an imprinted gene, we examined whether allelespecific expression changed after GNG12-AS1 depletion. DIRAS3 imprinting and its methylation status were unaltered (Supplementary Fig. $5 \mathrm{c}-\mathrm{e}$ ). Taken together, these results suggest that GNG12-AS1 modulates the expression of the already active DIRAS3 allele in cis and that siRNA to exon 1 of GNG12-AS1 disrupts this function without affecting expression of neighbouring genes.

siRNA against $5^{\prime}$ end of GNG12-AS1 blocks RNA Pol II. Next, we examined whether inhibition of DIRAS3 in cis can be achieved 
by targeting GNG12-AS1 exons closer to the DIRAS3 gene. Similar to when we targeted exon 7, siRNA to exon 5 efficiently reduced GNG12-AS1 (Supplementary Fig. 2b,c), but did not upregulate DIRAS3 (Supplementary Fig. 6a,b). In one of the cell lines, siRNA to exon 5 led to WLS downregulation. siRNAs to exons 2 and 3, which flank DIRAS3 on either side, reduced GNG12-AS1 isoform specifically (Supplementary Fig. 2f,g), suggesting that these siRNAs function post-transcriptionally. These siRNAs had no effect on DIRAS3 expression further supporting modulation via the act of transcription rather than through a specific GNG12-AS1 isoform (Supplementary Fig. 6c,d).
To prove that the cis function of GNG12-AS1 is modulated by siRNA targeting exon 1 , which leads to the inhibition of TI between GNG12-AS1 and DIRAS3, we profiled Pol II and histone modifications. As shown in Fig. 3a,b, a significant reduction in Pol II binding was observed at the TSS of GNG12-AS1 when exon 1, but not exon 7, was targeted by siRNA. Concomitantly, an increase in Pol II binding was observed at the DIRAS3 TSS. Nuclear run-on assays showed that the reduction in Pol II binding was associated with reduced nascent GNG12-AS1 transcription. Thus, a reduction of GNG12-AS1 and increased DIRAS3 run-on transcripts

a

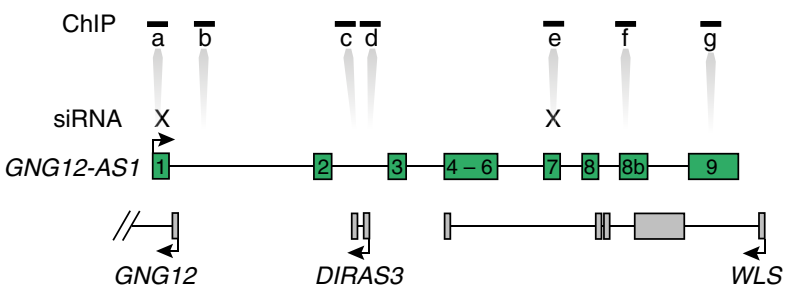

b

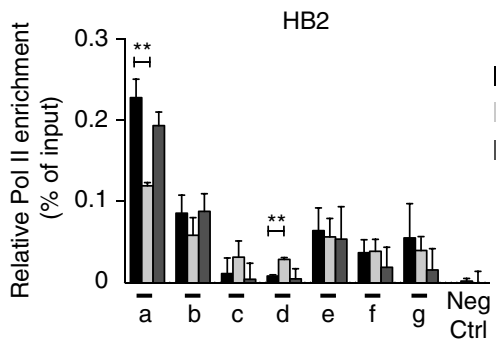

C
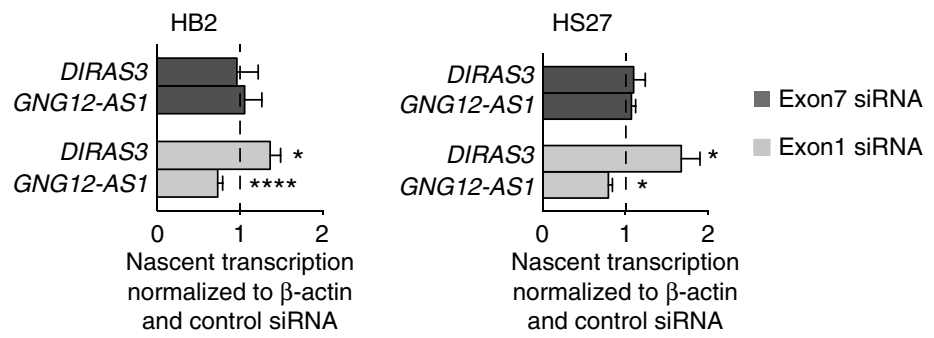

d

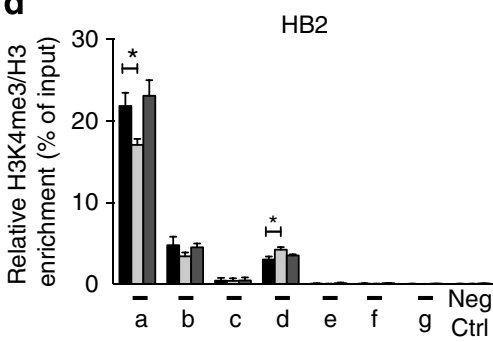

$\mathbf{e}$

Control siRNA

Exon1 siRNA

Exon7 siRNA

and control siRNA

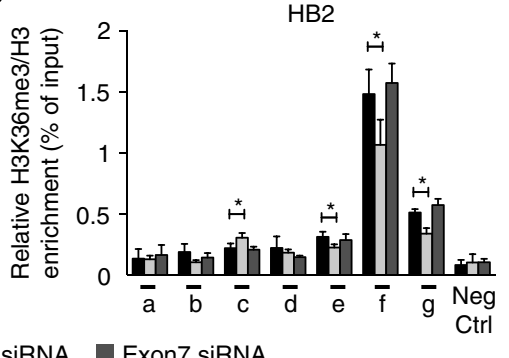

Figure 3 | Inhibition of Pol II after siRNA targeted to exon 1 of GNG12-AS1. (a) The GNG12-AS1 locus showing the location of primer sets (a-g) used for chromatin immunoprecipitation (ChIP) analysis. GNG12-AS1 TSS (a), region $1.6 \mathrm{~kb}$ downstream of GNG12-AS1 TSS (b), exon 2 of DIRAS3 (c), DIRAS3 TSS (d), GNG12-AS1 exon 7 (e), GNG12-AS1 exon 8b (f) and GNG12-AS1 exon 9 (g). The X indicates the exons where siRNAs were targeted. (b) Pol II ChIP analysis in HB2 cells treated with siRNA to exons 1 or 7 and controls. ChIP enrichments are presented as the percentage of protein bound, normalized to input. siRNA targeting exon 1 of GNG12-AS1, but not exon 7, results in reduced Pol II binding at GNG12-AS1 TSS (a). At the same time, increased Pol II binding was observed at the DIRAS3 TSS (d). (c) Nuclear run-on analysis of GNG12-AS1 and DIRAS3 transcription after treatment of HB2 cells (left panel) or HS27 cells (right panel) with control and exon 1 or exon 7 GNG12-AS1 siRNA. siRNA targeting exon 1 results in reduced GNG12-AS1 nascent transcription, which leads to increased DIRAS3 transcription. Data are normalized to $\beta$-actin and standardized to control siRNA, which is set up as 1 and shown as dotted line. (d,e) ChIP analysis from HB2 cells treated with siRNA to exons 1 or 7 using H3K4me3 (d) and H3K36me3 (e) antibodies. The precipitated DNA fragments were subjected to qRT-PCR analysis with the same primers as for Pol II ChIP. siRNA targeting exon 1, but not exon 7, of GNG12-AS1 reduced H3K4me3 at GNG12-AS1 TSS (a) and H3K36me3 levels in the body of GNG12-AS1 (e-g). Concomitantly, H3K4me3 and H3K36me3 levels increased at the DIRAS3 (d,c, respectively). Levels of H3K4me3 and H3K36me3 were normalized to histone $\mathrm{H} 3$ density. For all the graphs, ChIP enrichments are presented as the percentage of protein bound, normalized to input. Neg ctrl=negative control region. Error bars, s.e.m. $\left(n=3\right.$ biological replicates). ${ }^{\star} P<0.05$, ${ }^{\star \star} P<0.01$ and ${ }^{\star \star \star \star} P<0.0001$ by two-tailed Student's $t$-test. 
were observed with siRNA targeting exon 1, but not exon 7 (Fig. 3c).

To determine whether we could ablate unspliced transcripts and if so, whether this could affect DIRAS3 expression, we designed siRNAs to the first intron at 195 and $2,933 \mathrm{bp}$ downstream of the GNG12-AS1 TSS. In both HB2 and HS27 cell lines, these siRNAs resulted in a reduction of GNG12-AS1, indicating that the unspliced GNG12-AS1 transcript can be targeted by siRNA. However, increased DIRAS3 expression occurred only in HS27 cells (Supplementary Fig. 7a,d), indicating that siRNA specifically targeting exon 1 is more efficient at disrupting the regulatory relationship between GNG12-AS1 and DIRAS3 transcription.

Consistent with the changes in Pol II binding, we observed changes in active histone modifications when GNG12-AS1 was depleted using siRNA against exon 1, but not with exon 7 (Fig. 3d,e). Histone $\mathrm{H} 3$ lysine 4 trimethylation (H3K4me3) was reduced at the GNG12-AS1 TSS and increased at the DIRAS3 TSS. Furthermore, histone $\mathrm{H} 3$ lysine 36 trimethylation (H3K36me3), a marker for Pol II elongation ${ }^{39}$, was decreased in the body of GNG12-AS1 and increased within DIRAS3. As GNG12-AS1 depletion with exon 1 siRNA did not affect $W L S$ expression, a reduction in $\mathrm{H} 3 \mathrm{~K} 36 \mathrm{me} 3$ at $3^{\prime}$ end of GNG12-AS1 correlates with reduction in its transcriptional activity. The reduction of active histone modifications at the GNG12-AS1 TSS was not accompanied by a reciprocal increase of silencing modifications previously reported to be involved in TGS ${ }^{20,21,29}$, such as histone $\mathrm{H} 3$ lysine 9 trimethylation ( $\mathrm{H} 3 \mathrm{~K} 9 \mathrm{me} 3$ ) and histone $\mathrm{H} 3$ lysine 27 trimethylation (H3K27me3; Supplementary Fig. 8a,b). In addition, DNA methylation was unchanged at the GNG12-AS1 TSS (Supplementary Fig. 8c).

The changes in Pol II binding at the TSS of GNG12-AS1, together with the changes in active histone modifications associated with transcriptional activity after siRNA to exon 1, suggest that targeting the $5^{\prime}$ end of this lncRNA results in transcriptional silencing. However, to date, in all instances of TGS with exogenous siRNAs in human cells, the siRNAs were directed to the gene promoters. We therefore designed two siRNAs targeting GNG12-AS1 upstream of the TSS (33 and $129 \mathrm{bp}$ upstream of TSS). These siRNAs had no effect on GNG12-AS1 expression (Supplementary Fig. 7c,d), suggesting that transcription needs to be initiated for effective knockdown of the lncRNA.

In summary, siRNA targeting specifically exon 1 of GNG12-AS1 reduced its nascent transcription, without inducing changes in silent chromatin marks. Nevertheless, reduced transcription was associated with diminished Pol II activity and the redistribution of active histone marks, which led to increased DIRAS3 expression.

AGO2 mediates transcriptional inhibition of GNG12-AS1. As TGS is achieved through the RNAi machinery $24,25,27,28$, we investigated whether siRNA-mediated silencing of GNG12-AS1 involves Argonaute proteins. First, we examined whether the reduction in GNG12-AS1 transcript by siRNA targeted to exon 1 could be rescued by depletion of $A G O 1 / 2$ proteins. We found that depletion of AGO2 rescued GNG12-AS1 expression in cells exposed to siRNAs against exon 1 and exon 7 and abolished DIRAS3 upregulation in cells treated with siRNA against exon 1 (Fig. 4a). This effect was specific to AGO2, as AGO1 depletion did not rescue GNG12-AS1 depletion or DIRAS3 levels (Supplementary Fig. 9a). Similar results were obtained in the HS27 cell line (Supplementary Fig. 9b,c), confirming that AGO2 is involved in both the transcription- and post-transcriptionmediated mechanism of siRNA-directed downregulation of GNG12-AS1.

a
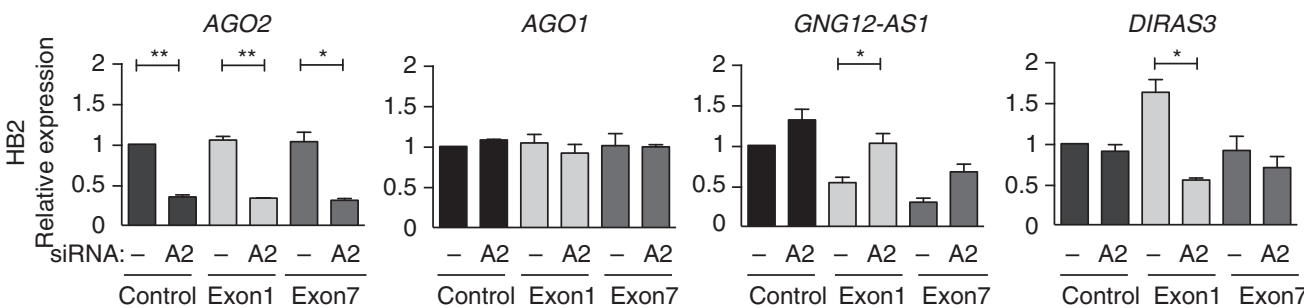

b

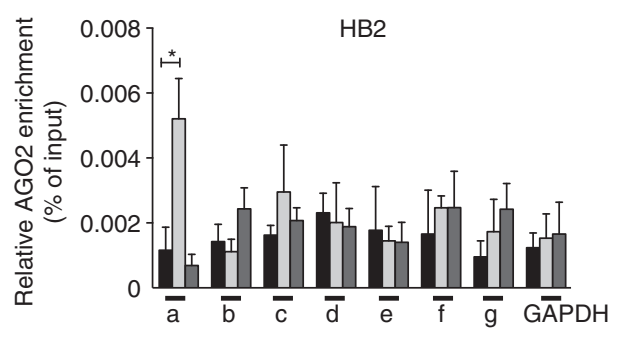

C

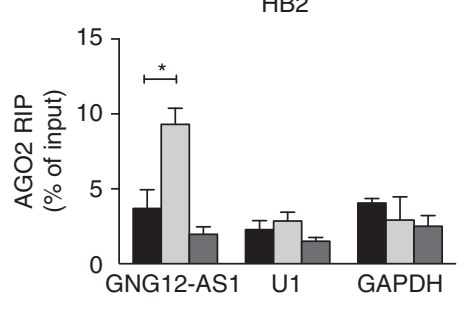

Control siRNA Exon1 siRNA Exon7 siRNA

Figure 4 | AGO2 mediates transcriptional inhibition of GNG12-AS1. (a) HB2 cells were transfected with siRNA to exon 1 or exon 7 of GNG12-AS1 together with control siRNA and siRNA targeting AGO2 (A2) (a) or AGO1 (A1) (Supplementary Fig. 9a). Expression levels of AGO2, AGO1, DIRAS3 and GNG12-AS1 were normalized to GAPDH and compared with control siRNA by qRT-PCR. AGO1 levels were not affected by either single AGO2 siRNA or double knockdown of AGO2 and GNG12-AS1. Transcriptional upregulation of DIRAS3 can be rescued by depletion of AGO2 and siRNA targeting exon 1 of GNG12AS1, whereas siRNA-mediated reduction of GNG12-AS1 with both siRNAs expression can be rescued by depletion of AGO2. (b) AGO2 ChIP analysis in HB2 cells after siRNA depletion of GNG12-AS1. The $x$ axis shows enrichment of AGO2 at regions described in Fig. 3a. AGO2 binding was enriched only at the TSS of GNG12-AS1 (a) after siRNA to exon 1. The GAPDH TSS was used as negative control region for AGO2 ChIP. (c) RIP from nuclear HB2 extracts showing association of AGO2 with GNG12-AS1 transcript after treatment of cells with exon 1 but not exon 7 siRNA. U1 and GAPDH were used as negative control RNAs for AGO2 RIP. RIP enrichments are presented as $\%$ of input RNA. For all the graphs, error bars indicate s.e.m. ( $n=3$ biological replicates). ${ }^{\star} P<0.05$ and ${ }^{\star *} P<0.01$ by two-tailed Student's $t$-test. 
We verified that $\mathrm{AGO} 2$ is present in the nucleus and cytoplasm (Supplementary Fig. 9d), as previously reported ${ }^{18}$. Next, we used chromatin immunoprecipitation (ChIP) analysis to see whether AGO2 associates with the GNG12-AS1 locus. We found that AGO2 was recruited to the GNG12-AS1 TSS only after treatment with siRNA directed to exon 1, but not exon 7 (Fig. 4b). This result suggests that AGO2 facilitates the physical interaction between exogenous siRNA to exon 1 and the chromatin to potentially mediate TGS of GNG12-AS1. Finally, we tested whether GNG12-AS1 associates with AGO2 in the nucleus. RNA immunoprecipitation (RIP) for AGO2 in nuclear extracts transfected with siRNAs showed an interaction between GNG12-AS1 and AGO2 only when siRNA targeted exon 1, and not exon 7 (Fig. 4c). Taken together, these results demonstrate that AGO2 mediates transcriptional silencing of GNG12-AS1 by siRNA complementary to exon 1 .

Cell cycle regulation by GNG12-AS1. DIRAS3 has been implicated in cell cycle regulation ${ }^{40}$, so we next asked if expression levels of DIRAS3 and GNG12-AS1 might correlate at different stages of the cell cycle. We synchronized cells with a double thymidine block (Fig. 5a), which arrest cells in S-phase and used cyclin E1 (G1/S transition marker) to monitor cell cycle progression (Fig. 5c). An increase in GNG12-AS1 transcription was observed after the release from double thymidine block, which coincided with the downregulation of DIRAS3. Cell cycle synchronization using serum starvation, followed by release with serum-containing media, showed similar results (Fig. 5b,d). The inverse relationship between expression levels of GNG12-AS1 and DIRAS3, which was tightly maintained throughout the cell cycle, further supports the evidence that GNG12-AS1 transcription modulates DIRAS3 expression in cis through TI.

We next examined the consequence of upregulating DIRAS3 after inhibiting GNG12-AS1. When GNG12-AS1 was depleted with exon 1 siRNA, we observed a small decrease in G1 cell number (control siRNA: $34 \%$ versus exon 1 siRNA: $29 \%$; $P=0.0575$; Fig. 5e,f). This result was confirmed by qRT-PCR and immunoblot analysis, which showed decreased cyclin E1 levels (Fig. $5 g-\mathrm{i}$ ). As expected, by combining siRNAs to DIRAS3 and exon 1 siRNA, we were able to neutralize the increase in DIRAS3 expression (Fig. 5g-i), which led to a modest but significant restoration of the G1 population (exon 1 siRNA: $29 \%$ versus exon 1/DIRAS3 siRNA: $36 \%, P=0.0105$; Fig. 5f). Analysis of cyclin $E 1$ levels showed that the reduction in G1 cell numbers could be partially rescued with the simultaneous depletion of GNG12-AS1 and DIRAS3 (Fig. 5g-i). By contrast, cyclin B1 levels did not differ among GNG12-AS1- and DIRAS3 siRNA-treated cells. Both RNA and protein levels of the cell cycle inhibitor $p 21$ increased when the cells were treated with siRNA to exon 1 in a DIRAS3- independent manner. S-phase distribution was unchanged after reducing GNG12-AS1. However, both exon 1 and exon 7 siRNAs increased the G2/M population (control siRNA: $23 \%$ versus exon 1 siRNA: $35 \%, P=0.0009$; or versus exon 7 siRNA: $30 \%, P=0.0126)$. As the increase in $\mathrm{G} 2 / \mathrm{M}$ population occurred upon simultaneous depletion of GNG12-AS1 and DIRAS3 (control siRNA: $23 \%$ versus exon 1/DIRAS3 siRNA: $29 \%, P=0.0169$; or versus exon 7/DIRAS3 siRNA: $30 \%$, $P=0.0084)$, we concluded that this $\mathrm{G} 2 / \mathrm{M}$ delay is a DIRAS3-independent event.

Taken together, these data indicate that GNG12-AS1 has a dual function in controlling cell cycle progression. Transcription of GNG12-AS1 influences the G1 phase of the cell cycle by modulating DIRAS3 expression, whereas the effect on G2/M phase of the cell cycle by GNG12-AS1 is a DIRAS3-independent event, and most likely represents the function of the RNA product.

GNG12-AS1 has a trans function regulating cell migration. DIRAS3 overexpression has been linked to cell migration and cell proliferation $^{32}$, so we assayed these cellular phenotypes after GNG12-AS1 knockdown. We found that reducing GNG12-AS1 with either of the siRNAs increased cell migration (Fig. 6a). As no difference was seen between the two siRNAs, we conclude that these phenotypic effects are due to the reduction of GNG12-AS1 transcripts and independent of DIRAS3. Indeed, a change in the migration phenotype was also observed in MCF7 cells, where DIRAS3 silencing cannot be reversed by GNG12-AS1 depletion (Fig. 6a). We next profiled global transcription changes after knockdown of GNG12-AS1 and found that both siRNAs against this lncRNA induced significant changes in cell adhesion and actin cytoskeleton pathways (Supplementary Fig. 10a,b). We focused on mesenchymal epithelial transition factor (MET), a tyrosine kinase receptor for the hepatocyte growth factor, known to regulate cell migration and invasion ${ }^{41,42}$ and found increased levels of MET (Fig. 6b,c and Supplementary Fig. 10c). In line with MET activation, we found elevated levels of the MET downstream target MAP2K4 (mitogen-activated protein kinase kinase 4$)^{42}$. This suggests that activation of MET signalling and its downstream targets could be responsible for the observed changes in cell migration. Interestingly, reducing specific minor isoforms of GNG12-AS1 containing exons 2 and 3 was sufficient to trigger activation of this signalling pathway. Future research into the secondary structure of the GNG12-AS1 will determine if this function is isoform specific. Finally, we examined whether simultaneously depleting GNG12-AS1 and DIRAS3 would prevent MET activation. Increased MET protein levels were maintained, confirming that GNG12-AS1 regulates MET signalling independently of DIRAS3 (Supplementary Fig. 10d). Altogether, these data indicate that the GNG12-AS1 transcript inhibits cell migration. In this context, GNG12-AS1 may have a tumoursuppressor function in addition to a role in modulating the expression levels of DIRAS3.

\section{Discussion}

LncRNAs can function via their RNA product and through the act of their transcription. So far it has been challenging to tease apart these two processes. Here we employed an siRNA-based strategy targeting different regions of GNG12-AS1 and found that siRNA selectively targeting the first exon of GNG12-AS1 resulted in increased expression of DIRAS3 in cis. These findings, together with inverse relationship between GNG12-AS1 and DIRAS3 during the cell cycle and Actinomycin $\mathrm{D}$ treatment, indicate that the act of transcribing GNG12-AS1 regulates expression of the active DIRAS3 allele by TI. We subsequently showed that this transcriptional targeting by siRNA to the $5^{\prime}$ end of GNG12-AS1 is mediated by AGO2, which binds to the GNG12-AS1 TSS as well as to its transcript. Finally, distinct functional consequences were uncovered, depending on whether GNG12-AS1 transcription was disrupted or whether its product was depleted.

The first suggestion that siRNA targeting exon 1 of GNG12-AS1 reduced its nascent transcription was with RNA-FISH, where we observed reduced hybridization with intronic probes after treating cells with this siRNA. More concrete biochemical evidence of transcriptional inhibition were demonstrated by decreased Pol II binding at the GNG12-AS1 TSS with ChIP and reduced nascent transcription with nuclear run-on experiments. siRNA targeted to exon 7, while still reducing the overall GNG12-AS1 product, does not upregulate DIRAS3 and 
a $\rightarrow$ GNG12-AS1 $\rightarrow$ DIRAS3

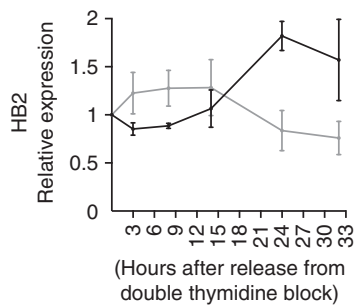

c

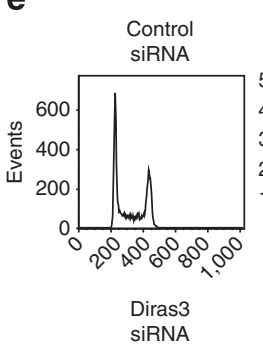

Release from

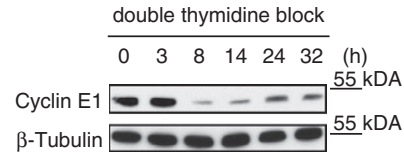

e

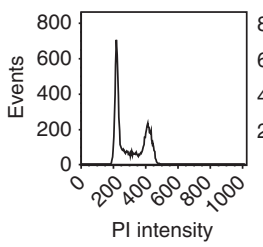

GNG12-AS1 exon1 siRNA
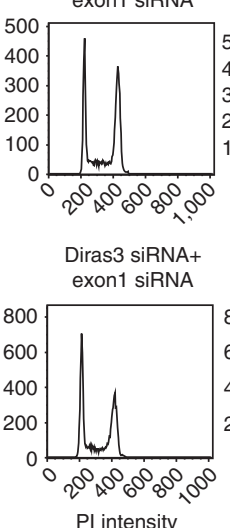

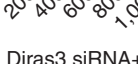

b $\rightarrow$ GNG12-AS1 $\rightarrow$ DIRAS3

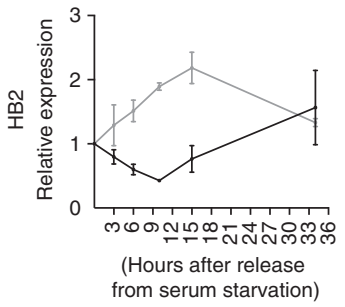

d

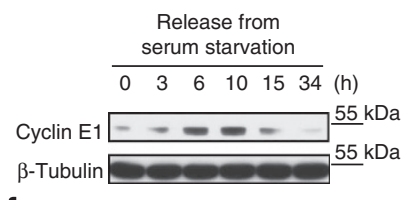

f

GNG12-AS1 exon7 siRNA
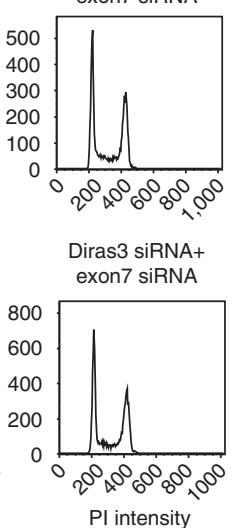

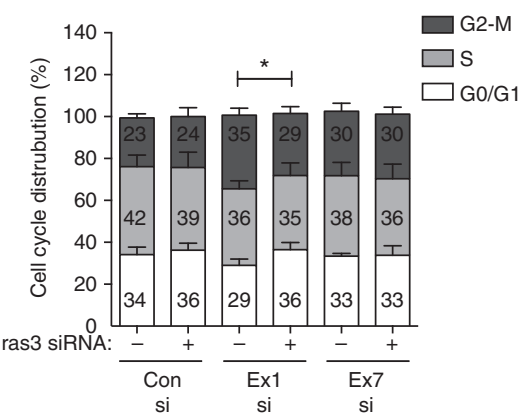

g

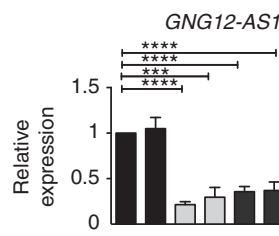

Diras3 siRNA:

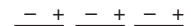
$\begin{array}{ccc}\text { Con } & \text { Ex1 } & \text { Ex7 } \\ \text { si } & \text { si } & \text { si }\end{array}$
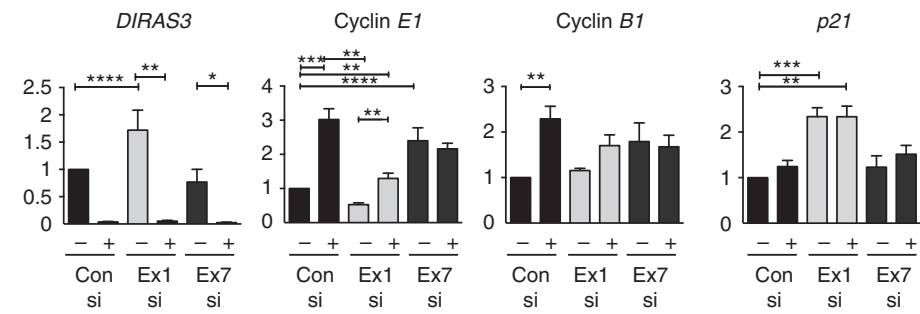

h

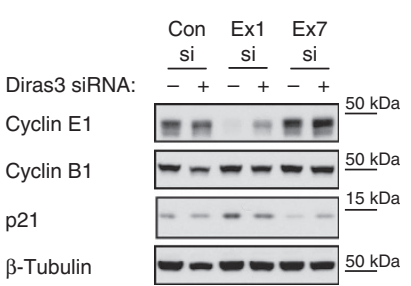

i
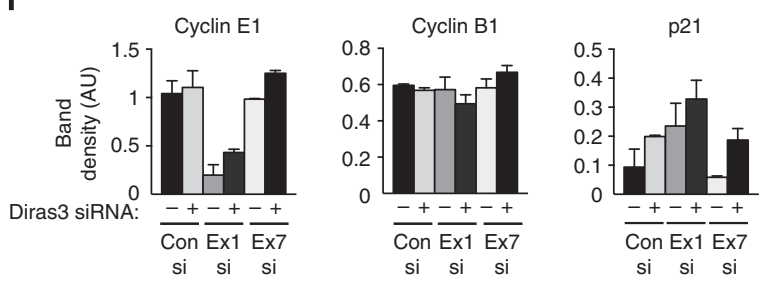

Figure 5 | DIRAS3-dependent and independent cell cycle regulation. (a,b) Expression patterns of GNG12-AS1 and DIRAS3 are inversely correlated in synchronized cells. HB2 cells were synchronized by double thymidine block (a) or serum starvation (b) and released at indicated time points. Expression levels were measured by qRT-PCR, normalized to GAPDH and standardized to time point $\mathrm{O}$ h (set as 1). Error bars, s.e.m. ( $n=3$ biological replicates). (c,d) Immunoblot of cyclin E1 (G1/S transition marker) was used to monitor cell cycle progression of HB2 cells synchronized with a double thymidine block (c) or serum starvation (d). $\beta$-Tubulin was used as a loading control. (e) FACS analysis of asynchronous HB2 cells after depletion of GNG12-AS1, DIRAS3 or simultaneous depletion of GNG12-AS1 and DIRAS3. The histograms are representative images of four biological replicates. (f) Cell cycle distribution of HB2 cells following siRNA conditions as shown in e. Note the restoration of G1 cell number between GNG12-AS1 exon 1 siRNA-treated cells and simultaneous depletion of GNG12-AS1 and DIRAS3 (control siRNA: 34\% versus exon 1 siRNA: $29 \%$, ${ }^{\star} P<0.05$ by two-tailed Student's $t$-test). Increase in G2/M population was observed between control and exon 1 siRNA (control siRNA: $23 \%$ versus exon 1 siRNA: $35 \%$, $\star \star \star ~ P<0.001)$ and exon 7 siRNA (control siRNA: $23 \%$ versus exon 7 siRNA: $30 \%$, ${ }^{\star} P<0.05$ by two-tailed Student's $t$-test). (g) qRT-PCR of DIRAS3, GNG12-AS1, cyclin E1, cyclin B1 and p21 after GNG12-AS1 and DIRAS3 depletion as shown in e. Expression levels are normalized to GAPDH and presented relative to control siRNA. Error bars, s.e.m. ( $n=4$ biological replicates). ${ }^{\star} P<0.05,{ }^{\star \star} P<0.01,{ }^{\star \star \star} P<0.001$ and ${ }^{\star \star \star \star} P<0.0001$ by two-tailed Student's $t$-test. (h) Immunoblot of cyclin E1, cyclin B1 and p21 after GNG12AS1 and DIRAS3 depletion in HB2 cells. Cyclin E1 was reduced only in exon 1 siRNA-treated cells and partially rescued after simultaneous depletion of GNG12-AS1 and DIRAS3 (compare lane 3 to lane 4). $\beta$-Tubulin was used as a loading control. (i) Quantification of immunoblots from (h; $n=2$ biological replicates). 
a

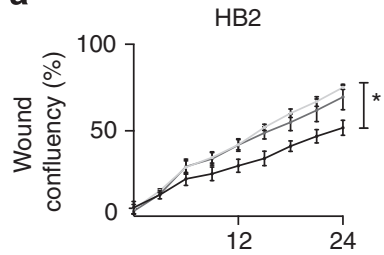

(Hours after wounding)

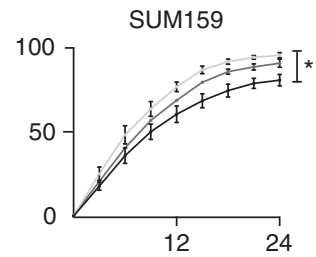

(Hours after wounding)

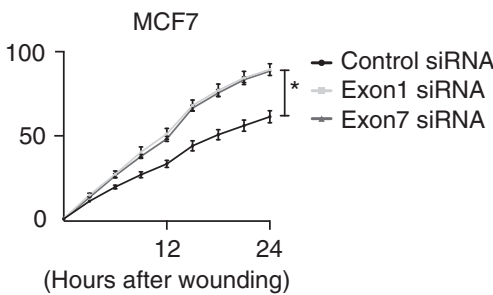

(Hours after wounding) b

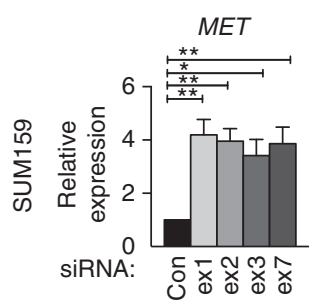

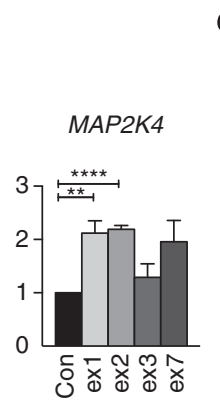

C

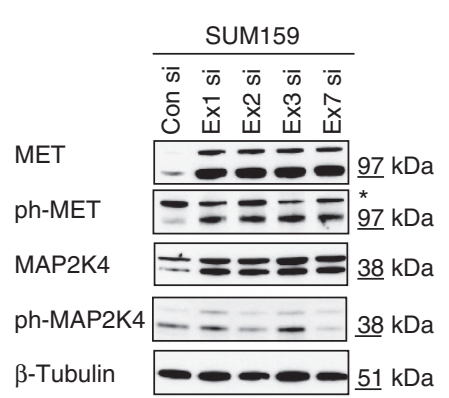

Figure 6 | GNG12-AS1 regulates cell migration independently of DIRAS3. (a) Cell migration is increased in HB2, SUM159 and MCF7 cells after GNG12-AS1 depletion. Cells were treated with control, exon 1 and exon 7 GNG12-AS1 siRNAs for $48 \mathrm{~h}$ before the wound-healing assay was performed over $24 \mathrm{~h}$ time course. Cells depleted with siRNA targeted to exon 1 and exon 7 of GNG12-AS1 have significantly increased cell migration. (b) Validation of gene expression changes in SUM159 cells after depletion of GNG12-AS1 with siRNAs targeting exons 1 and 7. Expression levels of MET and MAP2K4 were measured by qRT-PCR, normalized to GAPDH and compared with control siRNA. The knockdown efficiency of GNG12-AS1 is shown in Supplementary Fig. 2b,f.

(c) Immunoblot of MET, MAP2K4 and their active phosphorylated (ph) forms in SUM159 cells after depletion of GNG12-AS1 with siRNAs targeting exons 1 , 2, 3 and 7. Asterisk $\left({ }^{*}\right)$ indicates a non-specific band detected with an antibody specific to phosphorylated MET. $\beta$-Tubulin was used as a loading control. For all the graphs, error bars indicate s.e.m. ( $n=3$ biological replicates). ${ }^{\star} P<0.05,{ }^{\star \star} P<0.01$ and ${ }^{\star \star \star \star} P<0.0001$ by two-tailed Student's $t$-test.

does not change Pol II binding, making this siRNA an excellent internal control for our experiments.

Consistent with the data on Pol II binding and nascent transcription, we detected a reduction in the active histone modification, H3K4me3 at the GNG12-AS1 TSS when GNG12-AS1 was depleted using siRNA against exon 1, but not with exon 7. Similarly, the histone modification mark H3K36me3, which normally accumulates in gene body where it is associated with transcriptional elongation ${ }^{39}$, was reduced at the $3^{\prime}$ end of GNG12-AS1. Thus, our findings suggest that siRNA targeting the $5^{\prime}$ end of GNG12-AS1 led to inhibition of transcriptional initiation and elongation of GNG12-AS1 by directly interfering with Pol II dynamics without inducing changes in DNA methylation and repressive histone marks.

To date, all reported cases of exogenous TGS in human cells have used siRNAs against the gene promoter regions or the $\operatorname{TSS}^{17,20,24,25,29}$. Our study is, therefore, the first to show that targeting the first exon interferes with the transcription of a lncRNA. Indeed, with GNG12-AS1, targeting regions upstream of the TSS had no effect on its transcription, suggesting that transcription needs to be initiated in order to be targeted by siRNA. This may also explain why, although we found a reduction in active histone modifications, we did not observe an increase in repressive chromatin marks.

As TGS is achieved through the RNAi machinery $y^{24,25,27,28}$, we investigated the engagement of $\mathrm{AGO} 2$ in the inhibition of GNG12-AS1 transcription. siRNAs are known to form a complex with AGO2 in the cytoplasm and then shuffle between the cytoplasm and nucleus ${ }^{43}$. Small-RNA loading is a cytoplasmic process necessary for the nuclear import of AGO2 (refs 18,43,44). It has further been suggested that small RNAs can guide AGO2 to the promoters of ncRNAs and affect their transcription ${ }^{45,46}$. Based on these precedents, we would expect that all siRNAs in our experiments would have a similar knockdown efficiency in different cellular compartments, and once the AGO2-siRNA complex was in the nucleus, the functional consequences of the GNG12-AS1 depletion would depend on the region targeted. The presence of AGO2-siRNA complexes in the nucleus and cytoplasm would explain why we could rescue the GNG12-AS1 expression in double knockdown experiments between GNG12-AS1 and AGO2. However, AGO2-siRNA-GNG12-AS1 complexes at the GNG12-AS1 TSS could only be detected in the nucleus when the siRNA was directed to exon 1.

As described above, GNG12-AS1 could only be reduced when sequences downstream of its TSS were targeted by siRNA. This finding, together with the RIP results, showing that AGO2 associates with the GNG12-AS1 when cells are treated with siRNA to exon 1, suggest that transcription needs to have been already initiated for siRNA-mediated repression of transcription. Although AGO2-siRNA interactions with chromatin and the lncRNA could be demonstrated by ChIP and RIP, respectively, we do not have direct evidence for siRNA-AGO2 attaching the nascent lncRNA to the chromatin. We propose a model described in Fig. 7, where exogenous siRNA complementary to the $5^{\prime}$ end (exon 1) of GNG12-AS1 recruits AGO2 to GNG12-AS1 and to the chromatin at its TSS. This complex, containing the siRNA and GNG12-AS1 and possibly other proteins, stalls transcription of GNG12-AS1 by Pol II. DIRAS3 expression is enhanced as a consequence of reduced GNG12-AS1 transcription.

There are two possible mechanisms whereby an exogenous siRNA in a complex with $\mathrm{AGO} 2$ could lead to decreased GNG12-AS1 transcription. AGO2 has slicer activity ${ }^{47}$ and this could promote cleavage and degradation of the nascent GNG12-AS1. Alternatively, the AGO2-siRNA complex could present a physical block for Pol II initiation and elongation at the GNG12-AS1 TSS. Indeed, AGO2 has been shown to guide small RNAs to inhibit Pol II recruitment and elongation in human cells $^{24,48}$ independently of its slicer activity $24,27,45,46$. AGO2 has 

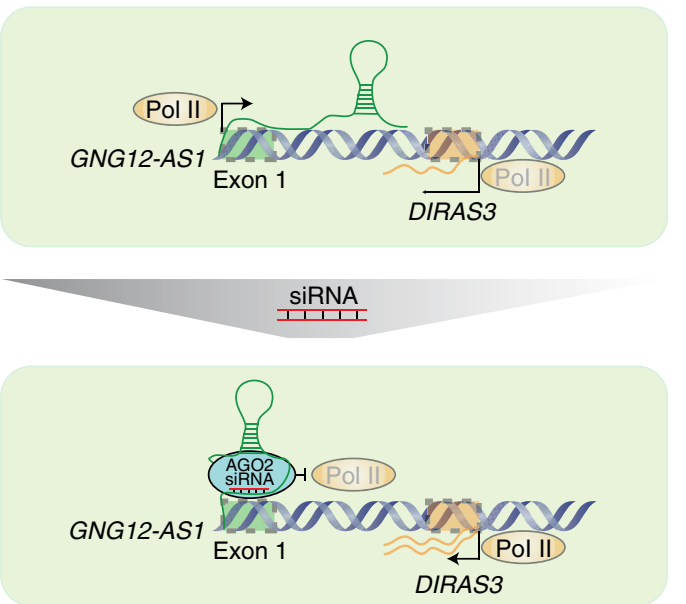

Figure 7 | Inhibition of transcriptional interference with siRNA. The top panel depicts how GNG12-AS1 modulates the expression of the active DIRAS3 allele through transcriptional interference. GNG12-AS1 may act as a rheostat for DIRAS3 transcription rate. The lower panel depicts how exogenous siRNA molecules in a complex with AGO2 can bind to both the TSS and GNG12-AS1 to inhibit Pol II, and block further transcription initiation and elongation of GNG12-AS1. As a result of transcriptional silencing of GNG12-AS1, transcriptional interference is reduced leading to increased transcription of DIRAS3.

been reported to form a complex with different chromatinmodifying proteins to mediate gene silencing ${ }^{49}$. It is therefore possible that AGO2-siRNA-GNG12-AS1 complexes at the TSS may include further proteins that influence Pol II kinetics. Our model does not distinguish between these different but not necessarily mutually exclusive mechanisms.

Silencing of imprinted genes is frequently achieved by TI of an antisense $\operatorname{lncRNA}{ }^{11,50}$. In these cases, the lncRNA is reciprocally imprinted relative to its target gene and is expressed on the opposite allele. Unlike, the other imprinted genes, we found that it was the already actively expressing DIRAS3 allele being upregulated after GNG12-AS1 knockdown, indicating that GNG12-AS1 has a role in modulating DIRAS3 expression in cis, rather than maintaining its imprinting. A reduction of Pol II and H3K4me3 at the TSS of GNG12-AS1 was associated with a concomitant increase at the DIRAS3 TSS. These shifts reflect the change in dynamics between these two genes as transcription inhibition by the GNG12-AS1 is eased and DIRAS3 transcription is augmented. TI between GNG12-AS1 and DIRAS3 could only be consistently inhibited when siRNAs were target within the first exon of GNG12-AS1. Targeting the adjacent intron did not lead to DIRAS3 upregulation in both of the cell lines tested. As GNG12-AS1 was efficiently reduced, we presume the nascent transcript was targeted, but GNG12-AS1 may also be present as mature unspliced transcripts that can be targeted posttranscriptionally. There are several enhancer signature peaks (histone H3 lysine 4 monomethylation (H3K4me1) and histone $\mathrm{H} 3$ lysine 27 acetylation (H3K27ac)) within this intron as annotated in the ENCODE database (Supplementary Fig. 11). It is possible that although the nascent RNA can be inhibited by siRNA targeted to this region, enough transcription has already occurred through the region to prevent DIRAS3 upregulation. In a region with multiple enhancers, some are likely to be tissue specific, which could explain why DIRAS3 expression is differentially upregulated in two different cell lines. The presence of enhancer marks at this locus would fit with GNG12-AS1 being an enhancer-like, cis regulator of DIRAS3 transcription ${ }^{51}$. The location of these enhancers and their potential specificity for DIRAS3 might explain why GNG12 is not affected by GNG12-AS1 depletion. Another possibility is that GNG12-AS1 expression is limited by GNG12 transcription and its further reduction has no impact on GNG12 expression.

An alternative strategy to siRNA for analysing the cis functions of GNG12-AS1 would have been to use CRISPRi, a new technology involving small guide RNAs to recruit a catalytically inactive Cas9 (dCas9) fused to the KRAB transcriptional repressor domain to specific loci ${ }^{52}$. Similar to RNAi, CRISPRi does not affect the underlying gene sequence and was shown to induce transcriptional repression of $\operatorname{lncRNAs}{ }^{52}$. However, KRABmediated silencing can spread to adjacent genes ${ }^{53}$, which would confound the detection of genes influenced by TI by a lncRNA.

Transcription of IncRNA provides an important mechanism for fine-tuning the expression of genes in cis $^{54}$. There is evidence for lncRNAs having a threshold-depended role in moderating gene regulation rather than an activating or repressive role ${ }^{54}$. For example, MALAT1, a lncRNA with several roles in gene regulation, has subtle effects on the expression of its neighbouring genes ${ }^{55}$. A fine-tuning mechanism could explain why small changes in gene expression observed after depletion of some lncRNAs could be relevant upon specific stress or environmental conditions. Imprinted genes are exquisitely regulated for gene dosage. It is likely that in vivo, the cis regulatory function of GNG12-AS1 in fine-tuning DIRAS3 expression is limited to specific developmental stages or environmental conditions as shown for HOTTIP $\operatorname{lncRNA}{ }^{56}$, which has similarly low abundance and expression levels. GNG12-AS1 is not imprinted in non-cancer cell lines, as shown previously by pyrosequencing SNP analysis ${ }^{31}$, but our RNA-FISH results indicate that in a substantial proportion of $\mathrm{HB} 2$ cells it may be randomly monoallelic. A growing number of autosomal genes are being shown to be randomly monoallelic at single-cell level ${ }^{57}$. If $50 \%$ of cells with GNG12-AS1 expression are expressing transcripts from the paternal allele, these would be repressing DIRAS3 in cis by TI, whereas on the other $50 \%$ of cells where GNG12-AS1 expression is maternal, DIRAS3 would already be silent on this allele. This would be consistent with the suggestion that random monoallelic expression refines gene expression levels in a population of cells ${ }^{58,59}$.

In addition to GNG12-AS1 having a cis function in moderating DIRAS3 transcription, GNG12-AS1 also regulates cell cycle progression. Transcriptional upregulation of DIRAS3 induced by GNG12-AS1 depletion led to G1 delay associated with the downregulation of cyclin E1. As the G1 delay and cyclin E1 levels were rescued by simultaneous depletion of exon 1 siRNA of GNG12-AS1 and DIRAS3, these data suggest that TI between GNG12-AS1 and DIRAS3 contributes to the regulation of G1 phase of the cell cycle. DIRAS3 was shown to be involved in cell cycle progression ${ }^{40,59}$. The upregulation of DIRAS3 by inhibiting GNG12-AS1 transcription in our study does not have the same effect on cyclin genes as previously reported, where DIRAS3 was ectopically overexpressed ${ }^{40,59,60}$. These differences could be due to the modest upregulation of DIRAS3 induced by transient repression of GNG12-AS1 with siRNA, whereas the latter study ectopically expressed DIRAS3. Nevertheless, our results are consistent with a role for DIRAS3 in cell cycle progression through G1 phase ${ }^{40,59}$. More importantly, these results indicate that disruption of TI between GNG12-AS1 and DIRAS3 can have effects on the cell cycle.

RNA-FISH analysis indicated that, in addition to co-localization between intronic and exonic probes, we also found cells in which the signals were separated, suggesting that there may be additional functions for GNG12-AS1. Our cell cycle analysis revealed that GNG12-AS1 depletion with siRNAs to $5^{\prime}$ end and $3^{\prime}$ end resulted in a G2/M delay. This effect is most likely due to a 
DIRAS3-independent function of GNG12-AS1 RNA product. This is further supported by our microarray experiments where both siRNAs induced changes in genes involved in cell cycle progression.

In addition, we found that reducing the levels of GNG12-AS1 transcripts whether by siRNAs directed to exon 1 or various other exons of GNG12-AS1 resulted in increased cell migration. This phenotype was observed upon GNG12-AS1 knockdown even in cells where DIRAS3 expression was absent. Expression profiling of GNG12-AS1-depleted cells showed an enrichment for genes involved in cell adhesion, migration and actin cytoskeleton pathways. We were able to validate the upregulation of MET signalling pathway after siRNA-mediated reduction of GNG12-AS1. MET controls many biological functions such as cell migration, invasion and motility, and its aberrant activation can lead to cancer $^{41,42,61}$. We have previously shown that GNG12-AS1 is downregulated in breast cancer tumours together with DIRAS3 (ref. 31). Thus, it is possible that GNG12-AS1 extends the tumoursuppressor function of this locus by regulating oncogenes such as MET. In keeping with this, MET was recently shown to be regulated by MEG3 lncRNA in pancreatic tumours ${ }^{62}$. Thus, MET could be a common target of IncRNAs involved in the regulation of cellular processes important for tumour growth. Further experiments are necessary to investigate whether the GNG12-AS1 transcript directly interacts with distant genes, and if so whether this interaction is isoform specific.

In summary, GNG12-AS1 is similar to several other lncRNAs that have both cis and trans functions ${ }^{63,64}$. Although the trans function may be to regulate genes involved in cell cycle and cell migration through direct interaction, the cis function has a role in fine-tuning regulation of DIRAS3 transcription. Altogether, our results demonstrate that an siRNA-based strategy can be employed to successfully separate functions that are due to lncRNA transcription from those of the transcript.

\section{Methods}

Cell culture and treatments. HB2 (human mammary epithelial cell line) were cultured in DMEM (GIBCO) supplemented with $10 \%$ fetal bovine serum (FBS), $10 \mathrm{mll}^{-1}$ penicillin-streptomycin solution, $5 \mathrm{mg} \mathrm{ml}^{-1}$ insulin (Sigma) and $1 \mathrm{mg} \mathrm{ml}^{-1}$ hydrocortisone (Sigma). MCF10A (human breast epithelial cell line) were cultured in MEGM SingleQuots (Lonza). SUM159 (breast cancer cell line) were cultured in Ham's F-12 medium (GIBCO) supplemented with 5\% FBS, $5 \mathrm{mg} \mathrm{ml}^{-1}$ insulin and $1 \mathrm{mg} \mathrm{ml}^{-1}$ hydrocortisone; MCF7 (breast cancer cell line) and HS27 (human foreskin fibroblasts) were cultured in DMEM with 10\% FBS and $10 \mathrm{mll}^{-1}$ penicillin-streptomycin solution. 293FT cells were grown in DMEM (high glucose $1 \times$; GIBCO, ref. 41965039) supplemented with $10 \%$ FBS, $0.1 \mathrm{mM}$ MEM Non-Essential Amino Acids (GIBCO), $6 \mathrm{mM}$ L-glutamine (GIBCO), $1 \mathrm{mM}$ MEM Sodium Pyruvate (GIBCO) and $500 \mu \mathrm{g} \mathrm{ml}^{-1}$ Geneticin (GIBCO). All the cells were from American Type Culture Collection and were cultured at $37^{\circ} \mathrm{C}$ with $5 \% \mathrm{CO}_{2}$. The cells were treated with Actinomycin-D (Sigma) at a final concentration of $10 \mu \mathrm{g} \mathrm{ml}^{-1}$ for $0,2,6,8$ and $24 \mathrm{~h}$.

Single-molecule RNA FISH. RNA FISH was performed as described ${ }^{37}$. Cells were grown on $18 \mathrm{~mm}$ round \# 1 cover glass, briefly washed with PBS and fixed with $\mathrm{PBS} / 3.7 \%$ formaldehyde at room temperature for $10 \mathrm{~min}$. Following fixation, cells were washed two times with PBS. The cells were then permeabilized in $70 \%$ ethanol for at least $1 \mathrm{~h}$ at $4{ }^{\circ} \mathrm{C}$. Stored cells were briefly rehydrated with Wash Buffer $(2 \times$ saline sodium citrate buffer (SSC), 10\% formamide) before FISH. The Stellaris FISH Probes (GNG12-AS1 intronic (Q670) and exonic probes (Q570), sequences in Supplementary Table 1) were added to the hybridization buffer $(2 \times$ SSC, $10 \%$ formamide, $10 \%$ dextran sulfate) at the same time to give a final concentration of $250 \mathrm{nM}$ per probe set. Hybridization was carried out in a humidified chamber at $37^{\circ} \mathrm{C}$ overnight. The following day, the cells were washed twice with Wash Buffer at $37^{\circ} \mathrm{C}$ for $30 \mathrm{~min}$ each. The second wash contained 4,6diamidino-2-phenylindole for nuclear staining $\left(5 \mathrm{ng} \mathrm{ml}^{-1}\right)$. The cells were then briefly washed with $2 \times$ SSC and then mounted in Vectashield (Vector Laboratories, H-1000). Images were captured using Nikon TE-2000 inverted microscope with NIS-elements software using a Plan Apochromat $\times 100$ objective and Andor Neo 5.5 sCMOS camera. We acquired 25 optical slices at $0.3 \mu \mathrm{m}$ intervals. Images were deconvolved with Huygens Professional and projected in two dimensions using Volocity Image Software Analysis. Intronic signals were scored to determine the percentage of cells with mono- or biallelic expression. Exonic signals were then scored on the same cells. To score whether intronic and exonic signals co-localize, we selected cells in which both signals were present.

Quantitative real-time PCR. RNA $(1 \mu \mathrm{g})$ was extracted with the RNeasy Kit (QIAGEN) and treated with DNase I (QIAGEN) following the manufacturer's instructions. QuantiTect Reverse Transcription Kit (QIAGEN) was used for cDNA synthesis including an additional step to eliminate genomic DNA contamination. Quantitative real-time PCR was performed on a 7900HT Fast Real-Time PCR System (Applied Biosystems) with Fast SYBR Green Master Mix (Life Technologies). Thermocycling parameters: $95^{\circ} \mathrm{C}$ for $20 \mathrm{~s}$ followed by 40 cycles of $95^{\circ} \mathrm{C}$ for $1 \mathrm{~s}$ and $60^{\circ} \mathrm{C}$ for $20 \mathrm{~s}$. Two reference genes (GAPDH and RPS18) were selected. Our microarray data show no significant variation in GAPDH and RPS18 expression in our siRNA experiments in HB2, HS27 and SUM159 cells. Expression levels of GNG12-AS1, DIRAS3, GNG12 and WLS in non-cancer and cancer cell lines are normalized relative to the geometric mean of GAPDH and RPS18 (Supplementary Fig. 1a). The expression levels using either GAPDH (Fig. 2c,d and Supplementary Fig. 3a,b) or the average of GAPDH and RPS18 (Supplementary Fig. 3c-f) across siRNA conditions were consistent and reproducible. Thus, we used only GAPDH normalization for further experiments. Exon 7-8 of GNG12-AS1 was used to quantify its expression, if not stated otherwise. To calculate the GNG12-AS1 copy number, standard curve of Ct values was performed by qRT-PCR using dilution series of known concentration of GNG12-AS1 DNA template (variant 1 that contains exon $1,2,3,4,5,6,7,8,8 \mathrm{~b}$ and 9). cDNA was made from RNA extracted from known number of different cell lines. The $\mathrm{Ct}$ values were fitted on the standard curve and the number of GNG12-AS1 molecules per cell was calculated. The final value was multiplied by 2 , to account for the fact that cDNA is single stranded and DNA templates used to make standard curve is double stranded. The sequences for expression primers are listed in Supplementary Table 2. The amplification efficiency of housekeeping genes was measured with serial dilution of cDNA of each gene from five different cell lines. PCR efficiencies and correlation coefficients (R2) for each primer pair are shown in Supplementary Table 3.

RNA interference. The sequences of siRNAs are present in the Supplementary Table 4. The cells were transfected with DharmaFECT 1 (SUM159, MCF7, MCF10A), DharmaFECT 3 (HS27) or DharmaFECT 4 (HB2) (Thermo-Scientific) following the manufacturer's instructions. All experiments were done $48 \mathrm{~h}$ after transfection and all the siRNAs were used at a final concentration of $50 \mathrm{nM}$.

siRNA-mediated depletion of GNG12-AS1. RNA and proteins were fractionated as described previously ${ }^{65}$. The cells were either untreated (for immunoblot analysis) or transfected with control and GNG12-AS1 siRNAs (for RNA analysis) RNA was isolated from cytoplasmic, nucleoplasmic and chromatin fractions by TRIZOL extraction (Life Technologies) and used for qRT-PCR. Data were normalized to the geometric mean of GAPDH and $\beta$-actin levels in each cellular compartment as no normalization controls is equal in all three compartments (for details see ref. 65). MALAT1 and RPS18 were used as positive controls for chromatin and cytoplasmic fractions, respectively. Primers used for this assay are listed in Supplementary Table 2.

Nuclear run-on assay. Nuclear run-on assay was performed as described previously ${ }^{66}$. Cells were grown in $10 \mathrm{~cm}$ dishes, trypsinized and centrifuged, and the pellets were washed with $1.5 \mathrm{ml}$ of NP-40 lysis buffer $(10 \mathrm{mM}$ Tris- $\mathrm{HCl}, 10 \mathrm{mM}$ $\mathrm{NaCl}, 3 \mathrm{mM} \mathrm{MgCl}, 0.5 \% \mathrm{NP}-40$ ), incubated on ice for $5 \mathrm{~min}$ and centrifuged at $1,500 \mathrm{~g}$ for $5 \mathrm{~min}$. Pellets were then washed again with $1.5 \mathrm{ml} \mathrm{NP}-40$ lysis buffer. The nuclei pellets were resuspended in $70 \mu \mathrm{l}$ glycerol storage buffer $(50 \mathrm{mM}$ Tris- $\mathrm{HCl}, \mathrm{pH} 8,0.1 \mathrm{mM}$ EDTA, $5 \mathrm{mM} \mathrm{MgCl}_{2}, 40 \%$ glycerol) and flash frozen in liquid nitrogen. $60 \mu \mathrm{l}$ were transferred to fresh nuclease-free tubes and equal amount of the $2 \times$ run-on transcription buffer $(20 \mathrm{mM}$ Tris- $\mathrm{HCl}, \mathrm{pH} 8 ; 5 \mathrm{mM}$ $\mathrm{MgCl}_{2}, 300 \mathrm{mM} \mathrm{KCl}, 4 \mathrm{mM}$ dithiothreitol) was added together with $2 \mathrm{mM}$ ATP, CTP, GTP (GE Healthcare) and $1 \mathrm{mM}$ Biotin-16-UTP (Epicentre). The reaction was incubated at $30^{\circ} \mathrm{C}$ for $45 \mathrm{~min}$. In vitro transcription was stopped by adding TURBO DNase (Ambion) at $37^{\circ} \mathrm{C}$ for $30 \mathrm{~min}$. Then $100 \mu \mathrm{l}$ of nuclei lysis buffer (50 mM Tris-HCl, pH 7.5; 5\% SDS, 0.125 M EDTA) and Proteinase K (Ambion) were added to the sample and incubated at $37^{\circ} \mathrm{C}$ for $30 \mathrm{~min}$. RNA extraction was performed using phenol/chloroform/isoamyl alcohol: 25:24:1 (Invitrogen) and chloroform/isoamyl alcohol: 24:1 (Sigma). To precipitate RNA, 3 M sodium acetate pH 5.5 (Life Technologies), GlycoBlue (Life Technologies) and ice-cold 100\% ethanol were added. The samples were incubated at $-80^{\circ} \mathrm{C}$ for $30 \mathrm{~min}$ and centrifuged at $12,000 \mathrm{~g}$ for $15 \mathrm{~min}$. Pellets were washed with ice-cold $70 \%$ ethanol and centrifuged at $12,000 \mathrm{~g}$ for $10 \mathrm{~min}$. The pellets were then resuspended in $30 \mu \mathrm{l}$ nuclease-free water. RNA samples were then purified on column using RNeasy kit (Qiagen) to remove free Biotin-16-UTPs, according to the manufacturer's instructions. Purified RNA from column was eluted in $30 \mu \mathrm{l}$ nuclease-free water. Biotinylated RNA from samples was captured using Dynabeads MyOne C1 streptavidin beads (Invitrogen). C1 beads ( $40 \mu \mathrm{l}$ per sample) were washed twice with Solution A ( $100 \mathrm{mM} \mathrm{NaOH}, 50 \mathrm{mM} \mathrm{NaCl})$ for 3 min and once with Solution B $(100 \mathrm{mM} \mathrm{NaCl})$. The $\mathrm{C} 1$ beads were then preblocked with BSA $\left(200 \mu \mathrm{g} \mathrm{ml}^{-1}\right)$ and Yeast tRNA $\left(200 \mu \mathrm{g} \mathrm{ml}^{-1}\right.$; Invitrogen) mix in Solution B. The beads were 
pre-blocked on a rotor at room temperature for $30 \mathrm{~min}$, collected on magnetic stand and resuspended in Solution B. Beads were aliquoted into nuclease-free tubes and equal amount $(20 \mu \mathrm{l})$ of RNA was added. Biotinylated RNA was pulled down for $2 \mathrm{~h}$ at room temperature. Then the beads were collected on magnetic stand, washed three times with $1 \times$ Wash/Binding buffer $(2 \times$ Wash/Binding buffer: $10 \mathrm{mM}$ Tris- $\mathrm{HCl}$, pH 7.4; $1 \mathrm{mM}$ EDTA, $2 \mathrm{M} \mathrm{NaCl}$ ). After the final wash, $\mathrm{C} 1$ beads with bound RNA were eluted in $30 \mu \mathrm{l}$ nuclease-free water and used directly for reverse transcription to prepare cDNA. Relative transcription was calculated as: $2^{\wedge}-[$ MeanCt(gene)-MeanCt( $\beta$-actin $\left.)\right]$. For GNG12-AS1 knockdown experiments, control transcription was set to 1. $\beta$-Actin primer was used for normalization (primers are listed in Supplementary Table 2).

\section{Lentivirus overexpression of GNG12-AS1 in human cells. The different} GNG12-AS1 splice variants and negative control vector (scrambled sequence) were first cloned in pJET1.2 plasmid (Fermentas) and then into modified pLenti6.3/TO/ V5-DEST vector (kindly provided by John Rinn, Harvard University) using Gateway cloning strategy. The sequences are listed in Supplementary Data 1. The modified vector does not contain WPRE, the SV40 promoter and the blasticidinresistance gene that could interfere with IncRNA structure and function. Sanger sequencing and restriction digestion using PvuI-HF and BsrGI enzymes confirmed the GNG12-AS1 inserts. Lentiviral transduction of GNG12-AS1 clones was done in 293FT cells using ViraPower (Invitrogen) including, negative and positive control vector containing mCherry. The DNA-Lipofectamine2000 complexes were added to 293FT cells and incubated overnight. Forty-eight and seventy-two hours post transfection, the virus-containing supernatants were harvested, centrifuged at $700 \mathrm{~g}$ for $5 \mathrm{~min}$ at $4{ }^{\circ} \mathrm{C}$ and filtered with $0.45 \mu \mathrm{m}$ with additional $0.22 \mu \mathrm{m}$ filter before being stored at $4{ }^{\circ} \mathrm{C}$. To transduce SUM159 cells, 80,000 cells per 12 -well plate were seeded in triplicate and transduced with the lentiviruses at a multiplicity of infection of 0.1 together with polybrene $(5 \mu \mathrm{g} \mathrm{ml}-1$, Sigma). Transduction efficiency ( $>80 \%$ ) was verified using positive control vector containing mCherry and measured by FACS Calibur Influx (Beckton Dickinson) using BDFACS Software 1.0.0.650. The FACS data were analysed using FlowJo software (TreeStar Inc). Exon 7-9 of GNG12-AS1 was used to quantify its overexpression (primers are listed in Supplementary Table 2).

Microarray analysis. Gene expression analysis was carried out on Illumina Human HT12 version 4 arrays. All data analyses were carried out on R using Bioconductor packages ${ }^{67}$. Raw intensity data from the array scanner were processed using the $\mathrm{BASH}^{68}$ and HULK algorithms as implemented in the bead array package ${ }^{69} . \log 2$ transformation and quantile normalization of the data were performed across all sample groups. Differential expression analysis was carried out using the Limma package ${ }^{70}$. Differentially expressed genes were selected using a $P$-value cutoff of $<0.05$ after application of FDR correction for multiple testing applied globally to correct for multiple contrasts. RNA was extracted from cells (HB2, SUM159) treated with control and GNG12-AS1 siRNAs (exons 1 and 7). The analysis was performed with six biological replicates for each cell line. cDNA synthesis, labelling and array procedure were conducted at the Genomic Facility at the CRUK CI. Pathway analysis was performed using Metacore.

MeDIP (methylated DNA immunoprecipitation) qRT-PCR. Genomic DNA was extracted from cells with control and GNG12-AS1 siRNA treated with $100 \mu \mathrm{g} \mathrm{ml}^{-1}$ RNaseA (Invitrogen) in $100 \mu \mathrm{l}$ of Tris-EDTA for $10 \mathrm{~min}$ at room temperature and sonicated for $5 \mathrm{~min}$ (Diagenode Picoruptor, $30 \mathrm{~s}$ ON-30sec OFF) to obtain an average fragments size of $300 \mathrm{bp}$. DNA was purified using 1.8 volumes AMPURE XP beads (Beckman Coulter) according to the manufacturer's instructions and resuspended in $185 \mu \mathrm{l}$ of water. The samples were boiled for $10 \mathrm{~min}$ and snap frozen on ice for $10 \mathrm{~min}$. Five microlitres were taken as input followed by addition of $20 \mu \mathrm{l}$ cold $10 \times$ meDIP buffer. Meanwhile, $20 \mu \mathrm{l}$ of Protein G Dynal beads (Invitrogen) were blocked with $\mathrm{PBS} / 0.01 \% \mathrm{BSA}$ for $30 \mathrm{~min}$ at room temperature and washed three times with $1 \times$ meDIP buffer. One microlitre of anti-5mC antibody (Diagenode) was conjugated to the washed beads in $200 \mu \mathrm{l}$ of $1 \times$ meDIP buffer for $2 \mathrm{~h}$ at room temperature. Antibody-bead conjugates were washed three times with cold $1 \times$ meDIP buffer, added to the sample DNA and incubated overnight at $4{ }^{\circ} \mathrm{C}$ in an overhead rotator. The bound material was washed three times with $200 \mu \mathrm{l}$ of $1 \times$ meDIP buffer at room temperature. Bound and input material were resuspended in $50 \mu \mathrm{l}$ lysis solution $(100 \mathrm{mM}$ Tris- $\mathrm{HCl}, \mathrm{pH} 5.5,5 \mathrm{mM}$ EDTA, $200 \mathrm{mM} \mathrm{NaCl}, 0.2 \%$ SDS plus $20 \mu \mathrm{g}$ proteinase K (Invitrogen 25530-049)) and incubated at $60^{\circ} \mathrm{C}$ for $30 \mathrm{~min}$. DNA was purified using 1.8 volumes AMPURE XP beads (Beckman Coulter) according to the manufacturer's instructions. Validation of Medip was done by qRT-PCR. The list of Medip primers is present in Supplementary Table 2.

Analysis of allelic expression and DNA methylation. Analysis of DNA methylation was by bisulfite conversion with the EZ DNA Methylation-Gold Kit (Zymo Research) and subsequent pyrosequencing as described ${ }^{31}$. The list of primers used for this experiment is present in Supplementary Table 5.
RNA immunoprecipitation. HB2 cells were transfected with control and GNG12-AS1 siRNAs and RIP was performed from nuclear extracts using mouse IgG (Cell Signaling) and AGO2 (Abcam) antibodies. Quality of cytoplasmic and nuclear extracts was assessed by immunoblot as described previously ${ }^{65}$. Eight micrograms of antibody were incubated with $70 \mu \mathrm{l}$ of Dynabeads Protein G beads (Life Technologies) in total volume of $280 \mu \mathrm{l}$ for $30 \mathrm{~min}$ at room temperature. The antibody-bead complex was incubated with $500 \mu \mathrm{g}$ of nuclear exactas for $2-3 \mathrm{~h}$ at $4{ }^{\circ} \mathrm{C}$ and then washed three times (5 min each) with equal amount of $1 \times$ NLB buffer (20 mM Tris-HCl, pH7.5, 0.15 M NaCl, $3 \mathrm{mM} \mathrm{MgCl}_{2}, 0.3 \%$ NP-40, $10 \%$ glycerol) supplemented with Complete EDTA free protease inhibitor cocktail (14549800; Roche) and phosphatase inhibitors ( $1 \mathrm{mM} \mathrm{NaF}, 1 \mathrm{mM} \mathrm{Na}_{3} \mathrm{VO}_{4}$ ) and RNAse OUT (100 U; Life Technologies). The RNA was extracted by addition of $1 \mathrm{ml}$ TRIzol (Life Technologies) to the beads, followed by $1 / 5$ volume of Chloroform (Sigma). 10\% of the input lysate was mixed with $1 \mathrm{ml}$ of TRIzol for the total input RNA. After centrifugation at $12,000 \mathrm{~g}$ for $15 \mathrm{~min}$ at $4{ }^{\circ} \mathrm{C}$, the aqueous supernatant was transferred to a new tube and RNA was precipitated with $1 / 10$ volume of $3 \mathrm{M}$ sodium acetate $\mathrm{pH} 5.5$ (Life Technologies), 1 volume of isopropanol (Sigma) and $1 \mu \mathrm{l}$ of GlycoBLue (Life Technologies) at $-80^{\circ} \mathrm{C}$ for $20 \mathrm{~min}$. After 30 min centrifugation at $12,000 \mathrm{~g}$ at $4^{\circ} \mathrm{C}$, the RNA pellet was washed twice with icecold $70 \%$ ethanol. Finally, the pellet was dissolved in $15 \mu \mathrm{l}$ of RNAse-free water (Life Technologies). We used Power SYBR Green RNA-to-CT 1-Step Kit (Life Technologies) for qRT-PCR. Primers for this assay were GNG12-AS1 (exons 7-8) and $U 1$ and GAPDH (negative controls for AGO2 binding). Primers are listed in Supplementary Table 2.

Cell lysis and immunoblot. Total cell lysis and immunoblot were performed as described previously ${ }^{31}$. Briefly, the cells were lysed in lysis buffer $(50 \mathrm{mM}$ Tris- $\mathrm{HCl}$, pH 8, 125 mM NaCl, 1\% NP-40, 2 mM EDTA, 1 mM phenylmethylsulphonyl fluoride, and protease inhibitor cocktail (Roche)) and incubated on ice for $25 \mathrm{~min}$. The proteins were denatured, reduced and separated using Nupage Novex 4-12\% Bis-Tris Protein Gels (Invitrogen). Secondary antibodies were conjugated with peroxidase, and immunobands were detected with a Supersignal West Dura HRP Detection Kit (Thermo-Scientific). Quantification of immunoblots was done on ImageScanner III (GE HealthCare) using the software package ImageQuant TL 7.0 (GE HealthCare). Uncropped scans of the immunoblots are shown in Supplementary Fig. 12.

Antibodies. The list of antibodies is present in Supplementary Table 6.

Chromatin immunoprecipitation. ChIP assays were performed as previously described ${ }^{31}$ with antibodies listed in the Supplementary Table 6. The input and the immunoprecipitated materials were quantified by QubitFluorometer (Life Technologies) with the dsDNA BR Assay Kit (Invitrogen). Thirty micrograms of chromatin and $5 \mu \mathrm{g}$ of antibody were used for ChIP experiment. The qPCR data were corrected for DNA amount, and enrichment was normalized against the input according to the formula $2-\mathrm{dCt}(\mathrm{Ab})-\log 2(\mathrm{DF})]-[\mathrm{Ct}($ input $)-\log 2(\mathrm{DF})]$. Primer sequences are present in Supplementary Table 2.

Cell synchronization. The HB2 cells were plated in six-well plates and $24 \mathrm{~h}$ later thymidine $(2 \mathrm{mM})$ was added. The cells were incubated for $18 \mathrm{~h}$, washed three times with PBS and released into thymidine-free medium for $9 \mathrm{~h}$. Thymidine was then added for a further $15 \mathrm{~h}$. The cells were then washed three times with PBS. At this point, the cells in G1/S phase were collected for RNA, FACS and protein analysis (T0) in a serum-rich medium without thymidine. Time points were collected 3 (T3), 8 (T8), 14 (T14), 24 (T24) and 32 (T32) hours later. In the case of serum starvation, HB2 cells were plated in six-well plates and then switched to serum-free medium for $48 \mathrm{~h}$. After starvation (T0), the cells were released into cell cycle by addition of serum and the time points for RNA and protein analysis were collected at 3 (T3), 6 (T6), 10 (T10), 15 (T15) and 34 (T34) hours. Cyclin E1 (G1/S transition marker) levels were used to monitor cell cycle progression by immunoblot. In the case of siRNA treatment, the cells were first transfected with siRNAs and thymidine was added $24 \mathrm{~h}$ later for additional $18 \mathrm{~h}$. The procedure was continued as described above.

Cell cycle analysis. The HB2 cells were transfected with siRNAs and harvested after $72 \mathrm{~h}$, washed with PBS and fixed with $70 \%$ ethanol (the samples were stored for up to 1 week at $4^{\circ} \mathrm{C}$ ). The cells were then washed once with PBS, incubated in PBS containing RNase A (100 $\mu \mathrm{g} \mathrm{ml}^{-1}$, Life Tecnologies) for $30 \mathrm{~min}$ at $37^{\circ} \mathrm{C}$, stained with propidium iodide $\left(20 \mu \mathrm{g} \mathrm{ml}^{-1}\right.$, Life Tecnologies) and incubated on ice in the dark for $30 \mathrm{~min}$. DNA content was analysed by FACS Calibur (Beckton Dickinson) using BD CellQuest Pro Software V6. DNA cell cycle analysis was performed on FlowJo software V9 (TreeStar Inc) to quantify cell cycle distribution.

Wound healing assay. Wound healing assay was performed on 24-well (Essen Imagelock) plates in triplicates after treatment of cells with control and siRNAs against GNG12-AS1. Forty-eight hours after siRNA treatment, scratch wounds 
were induced with $10 \mu \mathrm{l}$ sterile pipette tip, after which fresh culture medium was added. The IncuCyte 2011A Rev2 software was used to capture and analyse the pictures. The cell migration was followed in time for $24 \mathrm{~h}$ using IncuCyte FLR (Essen Bioscience), making measurements in triplicate every $3 \mathrm{~h}$.

Statistical analysis. The statistical significance of data was determined by twotailed Student's $t$-test in all experiments using GraphPad Prism unless indicated otherwise. $P$-values $>0.05$ were considered statistically not significant.

\section{References}

1. Rinn, J. L. \& Chang, H. Y. Genome regulation by long noncoding RNAs. Annu. Rev. Biochem. 81, 145-166 (2012).

2. Fatica, A. \& Bozzoni, I. Long non-coding RNAs: new players in cell differentiation and development. Nat. Rev. Genet. 15, 7-21 (2014).

3. Prensner, J. R. \& Chinnaiyan, A. M. The emergence of lncRNAs in cancer biology. Cancer Discov. 1, 391-407 (2011)

4. Kornienko, A. E., Guenzl, P. M., Barlow, D. P. \& Pauler, F. M. Gene regulation by the act of long non-coding RNA transcription. BMC Biol. 11, 59 (2013).

5. Bonasio, R. \& Shiekhattar, R. Regulation of transcription by long noncoding RNAs. Annu. Rev. Genet. 48, 433-455 (2014)

6. Shearwin, K. E., Callen, B. P. \& Egan, J. B. Transcriptional interference--a crash course. Trends Genet. 21, 339-345 (2005).

7. Pelechano, V. \& Steinmetz, L. M. Gene regulation by antisense transcription. Nat. Rev. Genet. 14, 880-893 (2013).

8. Petruk, S. et al. Transcription of bxd noncoding RNAs promoted by trithorax represses Ubx in cis by transcriptional interference. Cell 127, 1209-1221 (2006)

9. Hongay, C. F., Grisafi, P. L., Galitski, T. \& Fink, G. R. Antisense transcription controls cell fate in Saccharomyces cerevisiae. Cell 127, 735-745 (2006).

10. Bumgarner, S. L. et al. Single-cell analysis reveals that noncoding RNAs contribute to clonal heterogeneity by modulating transcription factor recruitment. Mol. Cell 45, 470-482 (2012).

11. Latos, P. A. et al. Airn transcriptional overlap, but not its lncRNA products, induces imprinted Igf2r silencing. Science 338, 1469-1472 (2012).

12. Martens, J. A., Laprade, L. \& Winston, F. Intergenic transcription is required to repress the Saccharomyces cerevisiae SER3 gene. Nature 429, 571-574 (2004).

13. Camblong, J., Iglesias, N., Fickentscher, C., Dieppois, G. \& Stutz, F. Antisense RNA stabilization induces transcriptional gene silencing via histone deacetylation in S. cerevisiae. Cell 131, 706-717 (2007)

14. Houseley, J., Rubbi, L., Grunstein, M., Tollervey, D. \& Vogelauer, M. A ncRNA modulates histone modification and mRNA induction in the yeast GAL gene cluster. Mol. Cell 32, 685-695 (2008).

15. Wilson, R. C. \& Doudna, J. A. Molecular mechanisms of RNA interference. Annu. Rev. Biophys. 42, 217-239 (2013).

16. Golding, M. C. et al. Depletion of Kcnq1otl non-coding RNA does not affect imprinting maintenance in stem cells. Development 138, 3667-3678 (2011).

17. Morris, K. V., Chan, S. W., Jacobsen, S. E. \& Looney, D. J. Small interfering RNA-induced transcriptional gene silencing in human cells. Science 305, 1289-1292 (2004).

18. Gagnon, K. T., Li, L., Chu, Y., Janowski, B. A. \& Corey, D. R. RNAi factors are present and active in human cell nuclei. Cell Rep. 6, 211-221 (2014).

19. Gagnon, K. T. \& Corey, D. R. Argonaute and the nuclear RNAs: new pathways for RNA-mediated control of gene expression. Nucleic Acid Ther. 22, 3-16 (2012)

20. Weinberg, M. S. et al. The antisense strand of small interfering RNAs directs histone methylation and transcriptional gene silencing in human cells. RNA 12, 256-262 (2006).

21. Hawkins, P. G., Santoso, S., Adams, C., Anest, V. \& Morris, K. V. Promoter targeted small RNAs induce long-term transcriptional gene silencing in human cells. Nucleic Acids Res. 37, 2984-2995 (2009).

22. Ting, A. H., Schuebel, K. E., Herman, J. G. \& Baylin, S. B. Short doublestranded RNA induces transcriptional gene silencing in human cancer cells in the absence of DNA methylation. Nat. Genet. 37, 906-910 (2005).

23. Janowski, B. A. et al. Inhibiting transcription of chromosomal DNA with antigene peptide nucleic acids. Nat. Chem. Biol. 1, 210-215 (2005).

24. Napoli, S., Pastori, C., Magistri, M., Carbone, G. M. \& Catapano, C. V. Promoter-specific transcriptional interference and c-myc gene silencing by siRNAs in human cells. EMBO J. 28, 1708-1719 (2009).

25. Jiang, G. et al. Small RNAs targeting transcription start site induce heparanase silencing through interference with transcription initiation in human cancer cells. PLoS ONE 7, e31379 (2012).

26. Morris, K. V., Santoso, S., Turner, A. M., Pastori, C. \& Hawkins, P. G. Bidirectional transcription directs both transcriptional gene activation and suppression in human cells. PLoS Genet. 4, e1000258 (2008).

27. Chu, Y., Yue, X., Younger, S. T., Janowski, B. A. \& Corey, D. R. Involvement of argonaute proteins in gene silencing and activation by RNAs complementary to a non-coding transcript at the progesterone receptor promoter. Nucleic Acids Res. 38, 7736-7748 (2010).
28. Janowski, B. A. et al. Involvement of AGO1 and AGO2 in mammalian transcriptional silencing. Nat. Struct. Mol. Biol. 13, 787-792 (2006).

29. Kim, D. H., Villeneuve, L. M., Morris, K. V. \& Rossi, J. J. Argonaute-1 directs siRNA-mediated transcriptional gene silencing in human cells. Nat. Struct. Mol. Biol. 13, 793-797 (2006)

30. Robb, G. B., Brown, K. M., Khurana, J. \& Rana, T. M. Specific and potent RNAi in the nucleus of human cells. Nat. Struct. Mol. Biol. 12, 133-137 (2005).

31. Niemczyk, M. et al. Imprinted chromatin around DIRAS3 regulates alternative splicing of GNG12-AS1, a long noncoding RNA. Am. J. Hum. Genet. 93, 224-235 (2013)

32. Yu, Y. et al. Biochemistry and biology of ARHI (DIRAS3), an imprinted tumor suppressor gene whose expression is lost in ovarian and breast cancers. Methods Enzymol. 407, 455-468 (2006).

33. Yu, Y. et al. NOEY2 (ARHI), an imprinted putative tumor suppressor gene in ovarian and breast carcinomas. Proc. Natl Acad. Sci. USA 96, 214-219 (1999).

34. Wang, L. et al. Loss of the expression of the tumor suppressor gene ARHI is associated with progression of breast cancer. Clin. Cancer Res. 9, 3660-3666 (2003).

35. Dalai, I. et al. Low expression of ARHI is associated with shorter progressionfree survival in pancreatic endocrine tumors. Neoplasia 9, 181-183 (2007).

36. West, J. A. et al. The long noncoding RNAs NEAT1 and MALAT1 bind active chromatin sites. Mol. Cell 55, 791-802 (2014).

37. Raj, A., van den Bogaard, P., Rifkin, S. A., van Oudenaarden, A. \& Tyagi, S. Imaging individual mRNA molecules using multiple singly labeled probes. Nat. Methods 5, 877-879 (2008).

38. Buehler, E., Chen, Y. C. \& Martin, S. C911: A bench-level control for sequence specific siRNA off-target effects. PLoS ONE 7, e51942 (2012).

39. Krogan, N. J. et al. Methylation of histone $\mathrm{H} 3$ by Set2 in Saccharomyces cerevisiae is linked to transcriptional elongation by RNA polymerase II. Mol. Cell Biol. 23, 4207-4218 (2003).

40. Li, Y. et al. Effects of ARHI on cell cycle progression and apoptosis levels of breast cancer cells. Tumour Biol. 33, 1403-1410 (2012).

41. Organ, S. L. \& Tsao, M. S. An overview of the c-MET signaling pathway. Ther Adv. Med. Oncol. 3, S7-S19 (2011).

42. Trusolino, L., Bertotti, A. \& Comoglio, P. M. MET signalling: principles and functions in development, organ regeneration and cancer. Nat. Rev. Mol. Cell Biol. 11, 834-848 (2010).

43. Ohrt, T. et al. Fluorescence correlation spectroscopy and fluorescence crosscorrelation spectroscopy reveal the cytoplasmic origination of loaded nuclear RISC in vivo in human cells. Nucleic Acids Res. 36, 6439-6449 (2008).

44. Schraivogel, D. \& Meister, G. Import routes and nuclear functions of Argonaute and other small RNA-silencing proteins. Trends Biochem. Sci. 39, 420-431 (2014).

45. Han, J., Kim, D. \& Morris, K. V. Promoter-associated RNA is required for RNA-directed transcriptional gene silencing in human cells. Proc. Natl Acad. Sci. USA 104, 12422-12427 (2007)

46. Schwartz, J. C. et al. Antisense transcripts are targets for activating small RNAs. Nat. Struct. Mol. Biol. 15, 842-848 (2008).

47. Meister, G. et al. Human Argonaute2 mediates RNA cleavage targeted by miRNAs and siRNAs. Mol. Cell. 15, 185-197 (2004).

48. Janowski, B. A. et al. Inhibiting gene expression at transcription start sites in chromosomal DNA with antigene RNAs. Nat. Chem. Biol. 1, 216-222 (2005).

49. Cho, S., Park, J. S. \& Kang, Y. K. AGO2 and SETDB1 cooperate in promotertargeted transcriptional silencing of the androgen receptor gene. Nucleic Acids Res. 42, 13545-13556 (2014).

50. Latos, P. A. \& Barlow, D. P. Regulation of imprinted expression by macro noncoding RNAs. RNA Biol. 6, 100-106 (2009).

51. Orom, U. A. \& Shiekhattar, R. Long non-coding RNAs and enhancers. Curr. Opin. Genet. Dev. 21, 194-198 (2011).

52. Gilbert, L. A. et al. Genome-scale CRISPR-mediated control of gene repression and activation. Cell 159, 647-661 (2014).

53. Groner, A. C. et al. KRAB-zinc finger proteins and KAP1 can mediate longrange transcriptional repression through heterochromatin spreading. PLoS Genet. 6, e1000869 (2010).

54. Maamar, H., Cabili, M. N., Rinn, J. \& Raj, A. linc-HOXA1 is a noncoding RNA that represses Hoxal transcription in cis. Genes Dev. 27, 1260-1271 (2013).

55. Zhang, B. et al. The IncRNA Malatl is dispensable for mouse development but its transcription plays a cis-regulatory role in the adult. Cell Rep. 2, 111-123 (2012).

56. Wang, K. C. et al. A long noncoding RNA maintains active chromatin to coordinate homeotic gene expression. Nature 472, 120-124 (2011).

57. Eckersley-Maslin, M. A. \& Spector, D. L. Random monoallelic expression: regulating gene expression one allele at a time. Trends Genet. 30, 237-244 (2014).

58. Gendrel, A. V. et al. Developmental dynamics and disease potential of random monoallelic gene expression. Dev. Cell 28, 366-380 (2014). 
59. Lu, X., Qian, J., Yu, Y., Yang, H. \& Li, J. Expression of the tumor suppressor ARHI inhibits the growth of pancreatic cancer cells by inducing G1 cell cycle arrest. Oncol. Rep. 22, 635-640 (2009).

60. Wu, X., Liang, L., Dong, L., Yu, Z. \& Fu, X. Effect of ARHI on lung cancer cell proliferation, apoptosis and invasion in vitro. Mol. Biol. Rep. 40, 2671-2678 (2013).

61. Gherardi, E., Birchmeier, W., Birchmeier, C. \& Vande Woude, G. Targeting MET in cancer: rationale and progress. Nat. Rev. Cancer 12, 89-103 (2012).

62. Modali, S. D., Parekh, V. I., Kebebew, E. \& Agarwal, S. K. Epigenetic regulation of the lncRNA MEG3 and its target c-MET in pancreatic neuroendocrine tumors. Mol. Endocrinol. 29, 224-237 (2015).

63. Takayama, K. et al. Androgen-responsive long noncoding RNA CTBP1-AS promotes prostate cancer. EMBO J. 32, 1665-1680 (2013).

64. Vance, K. W. et al. The long non-coding RNA Paupar regulates the expression of both local and distal genes. EMBO J. 33, 296-311 (2014).

65. Gagnon, K. T., Li, L., Janowski, B. A. \& Corey, D. R. Analysis of nuclear RNA interference in human cells by subcellular fractionation and Argonaute loading. Nat. Protoc. 9, 2045-2060 (2014).

66. Johnsson, P. et al. A pseudogene long-noncoding-RNA network regulates PTEN transcription and translation in human cells. Nat. Struct. Mol. Biol. 20, 440-446 (2013)

67. Gentleman, R. C. et al. Bioconductor: open software development for computational biology and bioinformatics. Genome Biol. 5, R80 (2004).

68. Cairns, J. M., Dunning, M. J., Ritchie, M. E., Russell, R. \& Lynch, A. G. BASH: a tool for managing BeadArray spatial artefacts. Bioinformatics 24, 2921-2922 (2008).

69. Dunning, M. J., Smith, M. L., Ritchie, M. E. \& Tavare, S. beadarray: R classes and methods for Illumina bead-based data. Bioinformatics 23, 2183-2184 (2007).

70. Smyth, G. K., Michaud, J. \& Scott, H. S. Use of within-array replicate spots for assessing differential expression in microarray experiments. Bioinformatics 21, 2067-2075 (2005).

\section{Acknowledgements}

We acknowledge all the members of Murrell, Rinn, Odom and Gergely laboratory as well as Massimiliano di Pietro, Klaas Mulder, Anna Git, Jason Carroll in Cambridge and Laurence Hurst (University of Bath) for reading and providing helpful comments on the manuscript. We also thank the Genomics, Microscopy and Bioinformatics core facilities at the Cambridge Institute, Christina Ernst for thumbnail image design, Ezgi Hacisuleyman for the design of the negative control vector, Cole Trapnell and David Hendrickson for providing us with lincExpress vector, Arjun Raj for help with the RNA FISH and Alaisdair Russell for help with the lentiviral work. This research was supported by the University of Cambridge, Cancer Research UK and Hutchison Whampoa Limited.

\section{Author contributions}

L.S., J.R., F.G. and A.M. conceived and designed the study. Data acquisition: L.S., M.N A.O., Y.I., A.E.M.R., N.J., S.W. and S.U-L. L.S., M.N., A.M., S.M., D.T.O. and F.G. performed the data analysis and interpreted the results. L.S. and A.M. with input from all of the authors wrote the manuscript.

\section{Additional information}

Accession codes: The microarray data have been deposited in the Gene Expression Omnibus of National Center for Biotechnology Information under the accession number GSE60563.

Supplementary Information accompanies this paper at http://www.nature.com/ naturecommunications

Competing financial interests: The authors declare no competing financial interests.

Reprints and permission information is available online at http://npg.nature.com/ reprintsandpermissions/

How to cite this article: Stojic, L. et al. Transcriptional silencing of long noncoding RNA GNG12-AS1 uncouples its transcriptional and product-related functions. Nat. Commun. 7:10406 doi: 10.1038/ncomms10406 (2016)

(c) (i) This work is licensed under a Creative Commons Attribution 4.0 International License. The images or other third party material in this article are included in the article's Creative Commons license, unless indicated otherwise in the credit line; if the material is not included under the Creative Commons license, users will need to obtain permission from the license holder to reproduce the material. To view a copy of this license, visit http://creativecommons.org/licenses/by/4.0/ 